\title{
High-mass X-ray binaries in the Small Magellanic Cloud ${ }^{\star}$
}

\author{
F. Haberl and R. Sturm
}

\author{
Max-Planck-Institut für extraterrestrische Physik, Giessenbachstraße, 85748 Garching, Germany \\ e-mail: fwh@mpe.mpg.de
}

Received 8 September 2015 / Accepted 28 October 2015

\begin{abstract}
Aims. The last comprehensive catalogue of high-mass X-ray binaries in the Small Magellanic Cloud (SMC) was published about ten years ago. Since then new such systems were discovered, mainly by X-ray observations with Chandra and XMM-Newton. For the majority of the proposed HMXBs in the SMC no X-ray pulsations were discovered as yet, and unless other properties of the X-ray source and/or the optical counterpart confirm their HMXB nature, they remain only candidate HMXBs.

Methods. From a literature search we collected a catalogue of 148 confirmed and candidate HMXBs in the SMC and investigated their properties to shed light on their real nature. Based on the sample of well-established HMXBs (the pulsars), we investigated which observed properties are most appropriate for a reliable classification. We defined different levels of confidence for a genuine HMXB based on spectral and temporal characteristics of the X-ray sources and colour-magnitude diagrams from the optical to the infrared of their likely counterparts. We also took the uncertainty in the X-ray position into account.

Results. We identify 27 objects that probably are misidentified because they lack an infrared excess of the proposed counterpart. They were mainly X-ray sources with a large positional uncertainty. This is supported by additional information obtained from more recent observations. Our catalogue comprises 121 relatively high-confidence HMXBs (the vast majority with Be companion stars). About half of the objects show X-ray pulsations, while for the rest no pulsations are known as yet. A comparison of the two subsamples suggests that long pulse periods in excess of a few $100 \mathrm{~s}$ are expected for the "non-pulsars", which are most likely undetected because of aperiodic variability on similar timescales and insufficiently long X-ray observations. The highest X-ray variability together with the lowest observed minimum fluxes for short-period pulsars indicate that in addition to the eccentricity of the orbit, its inclination against the plane of the Be star circum-stellar disc plays a major role in determining the outburst behaviour.

Conclusions. The large population of HMXBs in the SMC, in particular Be X-ray binaries, provides the largest homogeneous sample of such systems for statistical population studies.
\end{abstract}

Key words. Magellanic Clouds - galaxies: stellar content - stars: emission-line, Be - stars: neutron - X-rays: binaries - catalogs

\section{Introduction}

High-mass X-ray binaries (HMXBs) are comprised of an earlytype star and a compact object that orbit each other. The compact object is in most cases a neutron star (NS), but it can also be a black hole (see e.g. for the Magellanic Clouds, Liu et al. 2005) or a white dwarf (Sturm et al. 2012, and references therein). Many of the HMXBs show pulsations in their X-ray flux, which indicate the spin period of the NS. The Small Magellanic Cloud (SMC) is peculiar because it hosts exceptionally many known HMXBs. So far, the optical counterpart is only in one (well-confirmed) case a super-giant star (SMCX-1), while for all other identified cases a Be star (with Balmer emission lines) was found, forming a Be/X-ray binary (BeXRB). The last comprehensive catalogue of HMXBs in the SMC was published by Liu et al. (2005). In the meantime, new multiwavelength data were collected, and many new objects were found with Chandra, RXTE, Swift, and XMM-Newton observations of the SMC, which can at least be treated as candidates for this class of X-ray binaries. Most of the multi-wavelength work on the BeXRBs in the SMC concentrated on these pulsars (Coe \& Kirk 2015). In this work we present a complete list of known HMXBs in the SMC, including all sources that have been proposed at least as candidates in the literature. We

* The catalogue is only available at the CDS via anonymous ftp to cdsarc.u-strasbg.fr (130.79.128.5) or via

http://cdsarc.u-strasbg.fr/viz-bin/qcat?]/A+A/586/A81 use multi-wavelength information to identify some of the candidates as likely mis-identifications, and in our final catalogue we devise a scheme according to which the objects are a genuine HMXB system with different confidence levels.

\section{Catalogue}

For an updated catalogue of HMXBs and candidates in the SMC (and the Magellanic Bridge, which extends beyond the Eastern Wing) we compiled lists from the literature that present properties from large samples or searches for such systems (see Table 1) and include the more recent discoveries of individual objects. We collected information about the X-ray sources as well as their companion stars from the literature and included it in the catalogue to use it for statistical studies. An excerpt of our final catalogue is presented in Table A.1 (first the 63 pulsars sorted according to pulse period, followed by the other objects sorted by coordinates), and the full version is available at the CDS. The content is described in Table 2. In the comment column we provide key references for each source, which are listed in Table A.2.

\subsection{Special notes on catalogue sources}

The published lists of HMXBs in the SMC disagree in the details for some entries. In particular, sources with uncertain X-ray position or with a pulse period detected with low significance 
Table 1. Literature for HMXBs in the SMC.

\begin{tabular}{ll}
\hline \hline Reference & Description \\
\hline Liu et al. (2005) & HMXB catalogue of the Magellanic Clouds \\
Coe et al. (2005) & Optical properties of HMXBs in the SMC \\
Shtykovskiy \& Gilfanov (2005) & HMXBs in archival XMM-Newton data \\
McGowan et al. (2008b) & Chandra SMC Wing survey \\
Galache et al. (2008) & RXTE observations of SMC pulsars \\
McBride et al. (2008) & Optical spectroscopy of BeXRB pulsars in the SMC \\
Haberl et al. (2008) & New BeXRBs from XMM-Newton observations in 2006 and 2007 \\
Antoniou et al. (2009b) & Optical identification of Chandra sources \\
Antoniou et al. (2009a) & Optical spectroscopy of BeXRBs in the SMC \\
Laycock et al. (2010) & Catalogue of SMC sources from deep Chandra observations ${ }^{a}$ \\
Rajoelimanana et al. (2011b) & Long-term optical variability of HMXB pulsars in the SMC \\
Sturm et al. (2013c) & XMM-Newton survey of the SMC \\
Coe \& Kirk (2015) & BeXRB pulsars in the SMC \\
\hline
\end{tabular}

Notes. ${ }^{(a)}$ Includes detections down to two net counts. Most of these low-significance sources are not found in the Chandra Source Catalogue (CSC Evans et al. 2010).

can lead to different interpretations of their nature. The X-ray position of the majority of sources detected with Chandra or $X M M-N e w t o n$ is sufficiently accurate to uniquely identify their optical counterpart. However, there are cases whose angular separation exceeds $2-3 \sigma$ confidence, which cause the identification to be uncertain and increase the probability for a chance coincidence of the X-ray source with an early-type star. Moreover, the spin periods of pulsars evolve with time, and detections with an uncertain position of the X-ray source may not be associated uniquely with an object. In the following sections we provide information for such cases to introduce our classifications. In Sect. 2.1.3 we present a list of candidates that were previously rejected as HMXB and that we did not include in the catalogue.

\subsubsection{Update on individual BeXRB pulsars}

SXP7.92 - A new pulsar with a period of $7.92 \mathrm{~s}$ was discovered by (Corbet et al. 2008) in RXTE data. Coe et al. (2009) reported six detections of SXP7.92 with RXTE and suggested AzV285 as the probable optical counterpart. They detected an X-ray source using Swift consistently in position with AzV285. Israel et al. (2013) detected $7.92 \mathrm{~s}$ pulsations from a source at RA $=00: 57: 58.4$ and Dec $=-72: 22: 29.5$ (error 1.5") in Chandra data. The Chandra source is 20.4' away from AzV285, but is fully compatible with the RXTE pulsar. The source was probably also detected by ROSAT (RX J0057.9-7222, entry 75 in the HRI catalogue of Sasaki et al. 2000). We conclude that SXP7.92 = CXOU J005758.4-722229 = RX J0057.9-7222 is a BeXRB pulsar and the correct optical counterpart is 2MASS J00575856-7222290. This star shows outbursts every 40.03 days in OGLE II data, which is most likely the orbital period of the binary system (Schmidtke \& Cowley 2013). The detection of X-rays from the position of AzV285 with Swift suggests that this is the optical counterpart of another BeXRB, which is also confirmed by XMM-Newton observations (see below for XMM J010155.7-723236).

SXP9.13 - Pulsations with a period of $9.13 \mathrm{~s}$ were discovered from the ASCA source AX J0049-732 (Imanishi et al. 1998). The source is located in a crowded region of the SMC bar with two hard ROSAT sources (RXJ0049.5-7310 and RX J0049.2-7311) as possible counterparts (Filipović et al. 2000b). RX J0049.5-7310 was confirmed to be a BeXRB with a spin period of $894 \mathrm{~s}$ (Laycock et al. 2010). Consequently, several authors have alloted RX J0049.2-7311 to the $9.13 \mathrm{~s}$ pulsar.
However, RX J0049.2-7311 was covered by XMM-Newton and Chandra observations many times with sufficient photon statistics to expect a detection of the $9.13 \mathrm{~s}$ period. This was never seen, and therefore we do not identify RX J0049.2-7311 as the 9.13 s pulsar AX J0049-732, but instead keep two separate BeXRBs (within the ASCA error circle of $40^{\prime \prime}$ are $~ 25$ possible counterparts brighter than $V=18$ mag with $-0.2<B-V<0.2$ in the OGLE BVI photometric catalogue; Udalski et al. 1998).

SXP82.4 - XTE J0052-725 was discovered as a new transient in RXTE data (Corbet et al. 2002), and X-ray pulsations were found in archival Chandra observations. From the RXTE SMC monitoring Galache et al. (2008) identified an outburst pattern with a period of $362.3 \pm 4.1 \mathrm{~d}$ in X-rays. Rajoelimanana et al. (2011b) analysed the OGLE III data, which revealed a significant peak at $171 \pm 0.3 \mathrm{~d}$ in the power spectra, which is less than half the reported X-ray period. Galache et al. (2008) already noted that the X-ray period is longer than would be expected given its spin period position in the Corbet diagram (Corbet 1984; Laycock et al. 2005, see also Fig. 8, in which SXP82.4 is found as the right-most open red circle). We add here that the long X-ray period also places XTE J0052-725 outside the relation between $P_{\text {orb }}$ and the equivalent width of the $\mathrm{H} \alpha$ line (Reig et al. 1997, see also Fig. 9, in which SXP82.4 is again found as the right-most open red circle). This suggests that the orbital period might be closer to the period found in the optical.

SXP91.1 - Pulsations with 91 s were found in RXTE and ASCA data (XTE J0053-724 and AX J0051-722, Corbet et al. 1998). RXTE detections of pulsations at $85.4 \mathrm{~s}$ and $89.0 \mathrm{~s}$ were initially assigned to individual pulsars, but were later recognised as most likely stemming from one pulsar with a high spin-down rate (Galache et al. 2008; Corbet et al. 2010; Coe et al. 2011a). For our catalogue we only considered one pulsar (SXP91.1).

SXP4693 - The longest period detected from an SMC BeXRB of $4693 \mathrm{~s}$ was claimed by Laycock et al. (2010) from a $100 \mathrm{ks}$ Chandra observation with $\sim 140$ net source counts. The pulsar was covered by an observation of the XMM-Newton survey of the SMC on October 9-10, 2009 (ObsID 0601210801 with a net exposure of about $23 \mathrm{ks}$ and $\sim 1000$ net source counts; Sturm et al. 2013c). A Fourier analysis of the light curve reveals a peak at a frequency consistent with the suggested period. We investigated this further using folding techniques based on $\chi^{2}$ and Rayleigh $\mathrm{Z}^{2}$ tests (Buccheri et al. 1983) and a Bayesian periodic signal detection method (Gregory \& Loredo 1996) as described for instance in Coe et al. (2012). 
Table 2. Catalogue description.

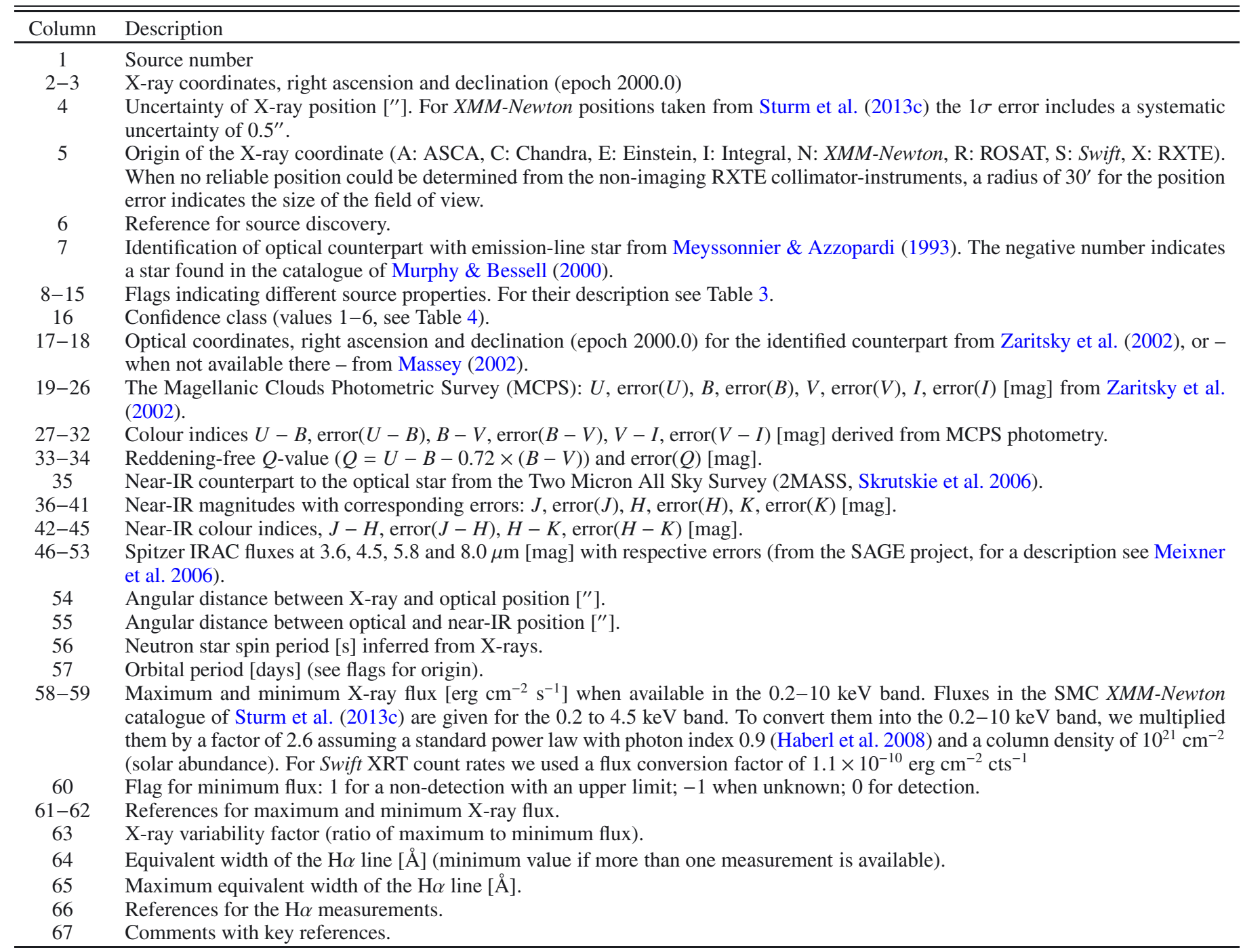

This resulted in a formal pulse period with $1 \sigma$ uncertainty of $4700 \pm 150 \mathrm{~s}$ (see Fig. 1). The light curve (Fig. 2) shows dips, which repeat every $\sim 4800 \mathrm{~s}$; they are most likely responsible for the periodicity seen in the timing analysis. To confirm that this pattern is strictly periodic requires a longer observation, but we conclude that the XMM-Newton observation finds evidence for a period around $4800 \mathrm{~s}$ in agreement with the Chandra results presented by Laycock et al. (2010). Coe \& Kirk (2015) listed the pulsar twice with two different periods as SXP6.62 and SXP4693. We do not see a significant signal near $6.62 \mathrm{~s}$ in the XMM-Newton data.

\subsubsection{Sources without detected pulsations}

RX J0032.9-7348 - This hard and variable ROSAT source was discovered by Kahabka \& Pietsch (1996) in PSPC observations and proposed as HMXB candidate. Stevens et al. (1999) found two early-type stars in the ROSAT error circle, one of them showing $\mathrm{H} \alpha$ emission (their object 1). Object 2 was identified with GSC0914101338, for which (Evans et al. 2004) give a spectral type of B0.5V. Object 2 is brighter $(B=15.50 \mathrm{mag}$, $V=15.24$ ) and slightly more distant to the best PSPC position (Haberl \& Sasaki 2000). Although object 2 cannot be completely ruled out, we assume object 1 as optical counterpart of the X-ray source because of its measured $\mathrm{H} \alpha$ emission line, which suggests a BeXRB nature, but we flag the optical identification as uncertain.

RX J0045.6-7313 - Haberl \& Sasaki (2000) suggested this ROSAT source as a BeXRB candidate because an emissionline star is located in the X-ray error circle (object 114 in Meyssonnier \& Azzopardi 1993). More recent catalogues of early-type stars in the SMC contain additional possible counterpart candidates: Bonanos et al. (2010) performed IR photometry of massive stars and listed AzV9 (Azzopardi et al. 1975) with spectral class B0III (UV spectral classification by Smith Neubig \& Bruhweiler 1997) and IR colours consistent with that of a Be star (see Sect. 3 and Fig. 4). We add AzV9 to our catalogue and flag the optical identification as uncertain.

CXOU J004941.43-724843.8 - This source was detected in the Chandra survey of the SMC bar (Antoniou et al. 2009b, source ID 7_19) with the proposed optical counterpart $(V=$ $17.17 \mathrm{mag}$ ) 1.36" away from the X-ray position. With an X-ray luminosity of $3.7 \times 10^{33} \mathrm{erg} \mathrm{s}^{-1}$ the source was at low luminosity during the Chandra observation (Maravelias et al. 2014), and it was not detected by XMM-Newton (Sturm et al. 2013c). Maravelias et al. (2014) presented spectroscopy of the optical star and reported the detection of a narrow $\mathrm{H} \alpha$ line, concluding on a spectral type B1-B5 but uncertain Be nature because 


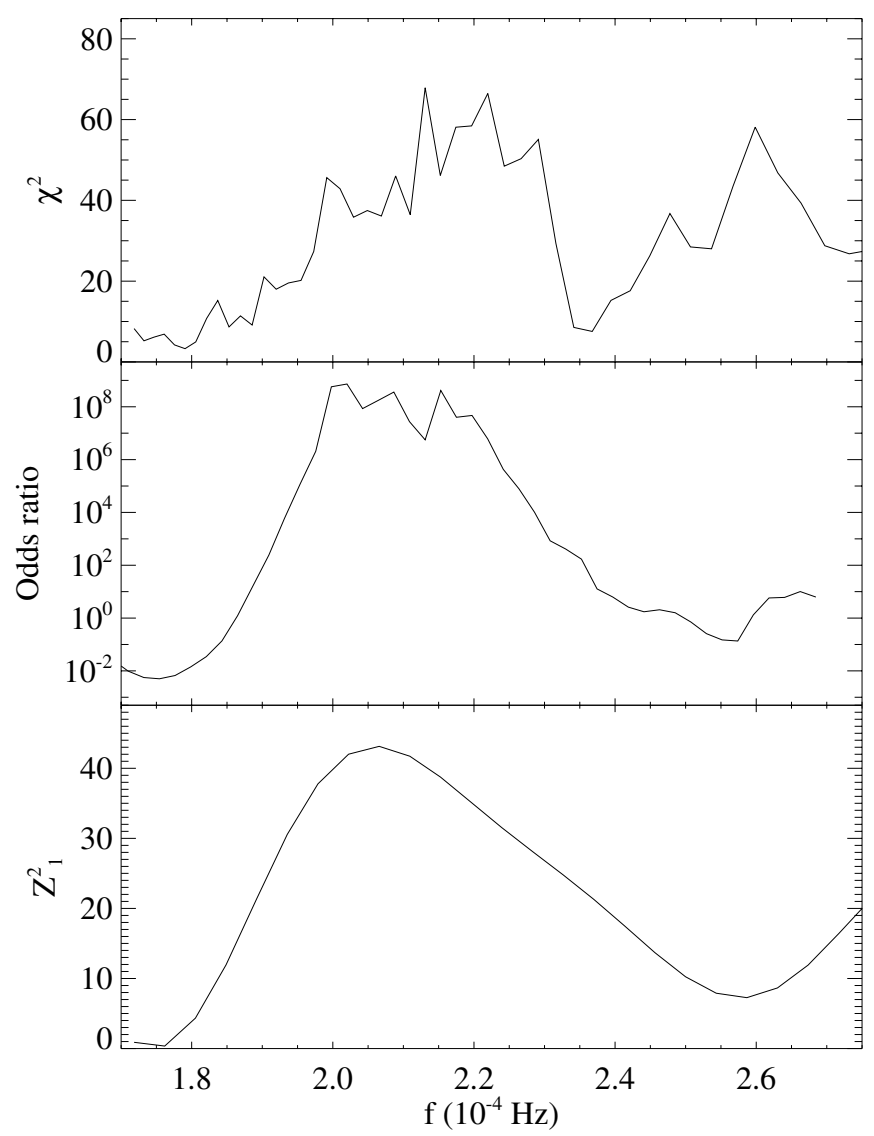

Fig. 1. Periodograms obtained from the combined EPIC data of SXP4693 from the XMM-Newton observation 0601210801 $(0.2-10 \mathrm{keV}) \cdot \chi^{2}$ test, Bayesian odds ratio, and Rayleigh $\mathrm{Z}_{1}^{2}$ test are shown from top to bottom.

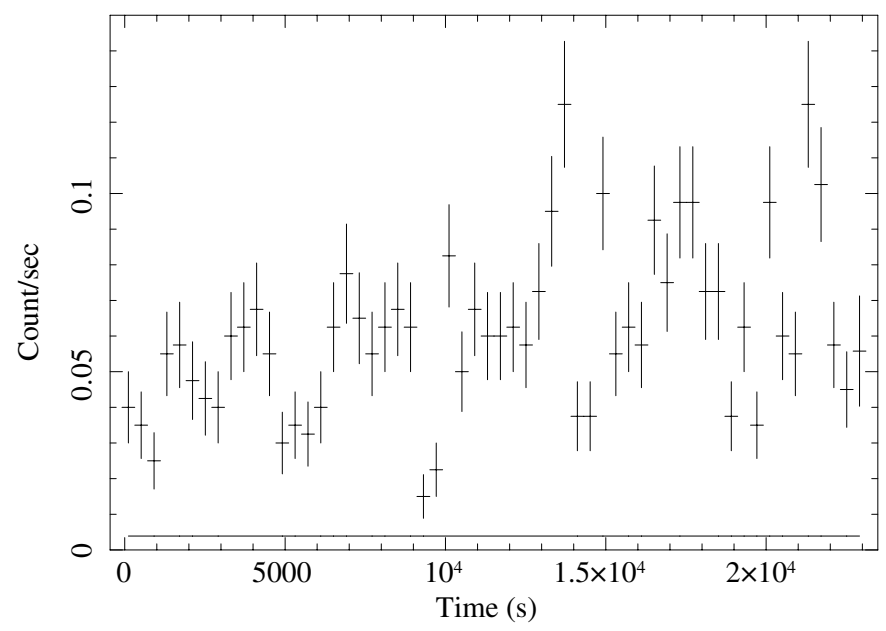

Fig. 2. EPIC light curve $(0.2-10 \mathrm{keV})$ of SXP4963 with a binning of $400 \mathrm{~s}$. The horizontal line at $3.9 \times 10^{-3} \mathrm{cts} \mathrm{s}^{-1}$ marks the background level.

of the marginal line width. All these ambiguities make this case inconclusive.

XMMU J005723.4-722356 - Shtykovskiy \& Gilfanov (2005) proposed the source detected in XMM-Newton data as an HMXB candidate because it might be associated with an early-type star. The spectral type of the proposed counterpart was given as B2 (II) by Evans et al. (2004) and confirmed by Maravelias et al. (2014). In the SMC catalogue (source 65) of Sturm et al. (2013c), the XMM-Newton position is $1.73^{\prime \prime}$ offset from the optical position given by Zaritsky et al. (2002). Similarly, the Chandra position is $1.3^{\prime \prime}$ away (Antoniou et al. 2009b; Maravelias et al. 2014). Both X-ray positions are more consistent with that of an AGN (Sturm et al. 2013c). The source was also detected in three recent XMM-Newton observations of SXP5.05 (Coe et al. 2015) at a much brighter level than in the past. During the last observation (ID 0700580601) in particular, with $2.4 \times 10^{-13} \mathrm{erg} \mathrm{cm}^{-2} \mathrm{~s}^{-1}$ the flux was a factor of $\sim 5$ higher than the previous maximum during observation 0084200101. Using the upper limit from observation 0500980201 of $5.8 \times 10^{-15} \mathrm{erg} \mathrm{cm}^{-2} \mathrm{~s}^{-1}$, we derive a variability factor of at least $\sim 40$, which strongly favours an HMXB. The X-ray position in all three new observations also agrees better with the B2 star (distances between $0.45^{\prime \prime}$ and $1.0^{\prime \prime}$ ), which is further improved when using the target of the observations, SXP5.05 and its optical counterpart, for bore-sight corrections (resulting in distances of between $0.36^{\prime \prime}$ and $0.68^{\prime \prime}$ ). Optical identification with the early-type star and the strong X-ray variability suggest that XMMU J005723.4-722356 is an HMXB.

XMM J010155.7-723236 - Source number 816 in the SMC catalogue of Sturm et al. (2013c) was originally assigned to SXP7.92. However, no pulsations were detected in the $X M M-N e w t o n$ data. The improved XMM-Newton position is consistent with the Swift source detected near AzV285 (see SXP7.92 above) and confirms this star as the optical counterpart. A flux upper limit from one XMM-Newton observation with no detection of the source combined with the Swift flux yields a flux ratio of $>900$, clearly suggesting a BeXRB nature of the source. Analysing OGLE II and III data of AzV285, (Coe et al. 2009) found a 36.79 d period, which was revised by Rajoelimanana et al. (2011b) to $36.41 \pm 0.02 \mathrm{~d}$; this is probably the binary period.

XMMU J010429.4-723136 - Source number 3285 in the SMC catalogue of Sturm et al. (2013c) was proposed by these authors as an HMXB candidate. The large X-ray variability and the brightness of the proposed optical counterpart indicates a BeXRB nature of the source. The source is most likely identical to the Chandra source CXOU J010428.7-723134 (Rajoelimanana et al. 2011a; Schmidtke et al. 2013a), although the Chandra position is $2.8^{\prime \prime}$ away from that of the proposed counterpart (using the coordinates from Zaritsky et al. 2002). The optical star shows a period of 37.15 days in OGLE and MACHO data (Rajoelimanana et al. 2011a), which might indicate the orbital period, but could also be an alias of a $0.972 \mathrm{~d}$ period, which Schmidtke et al. (2013a) interpreted as non-radial pulsations of a Be star. Rajoelimanana et al. (2011a) also reported a period of $707 \mathrm{~s}$ found in the Chandra X-ray data. However, this period is most likely an instrumental effect: it is exactly the Chandra dithering period and the source is located at the rim of a CCD, moving in and out of the detector. This probably also explains the relatively large angular distance between the Chandra and the optical position. A likely ROSAT detection ([HFP2000]264) is listed in the PSPC catalogue of Haberl et al. (2000). We conclude that XMMU J010429.4-723136 = CXOU J010428.7-723134 = RX J0104.5-7231 is a BeXRB in the SMC, although measurements of the spin period of the NS and of the strength of the $\mathrm{H} \alpha$ line are still required for the final confirmation.

\subsubsection{Rejected candidates from previous work}

For completeness we provide here a list of former HMXB candidates, which were rejected in earlier work. These objects are not included in our catalogue. 
XMMU J004833.4-732355 - Source number 40 (uncertain nature) in Table 2 of Shtykovskiy \& Gilfanov (2005) is classified as an AGN by Sturm et al. (2013c). See source 123 in their catalogue.

XMMU J005156.0-734151 = RXJ0051.7-7341 - Source number 1 of Sasaki et al. (2003) is rejected as an HMXB candidate. In the improved error circle of the source derived from additional observations (source 100 in the SMC catalogue of Sturm et al. 2013c) no bright optical counterpart with colours of an early-type star is found.

XMMU J005432.2-721809 - Source number 36 (uncertain nature) in Table 2 of Shtykovskiy \& Gilfanov (2005) is newly classified as AGN (source 62 in Sturm et al. 2013c). From the $\mathrm{X}$-ray spectrum a photon index typical for an AGN is derived and the position is $2.7^{\prime \prime}(4.8 \sigma)$ away from the $V=16.6 \mathrm{mag}$ star, originally proposed as the counterpart.

XMMU J005441.1-721720 - Another source of uncertain nature (number 41) from Shtykovskiy \& Gilfanov (2005). The revised position (source 82 in Sturm et al. 2013c) is inconsistent with the originally proposed counterpart ( $V=15.7$ mag star), and the source is classified as AGN candidate.

CXOU J005504.40-722230.4 - Maravelias et al. (2014) presented optical spectroscopy of a B star 3.6" away from the Chandra position. The large angular distance and the faintness ( $V=17.86 \mathrm{mag})$ of this star make a misidentification very likely. Moreover, from the XMM-Newton detection of the X-ray source (source 976 in Sturm et al. 2013c) the most likely counterpart is classified as AGN using a mid-IR colour selection (Kozłowski \& Kochanek 2009).

CXOUJ005527.9-721058 was found in Chandra data and suggested as a BeXRB pulsar by Edge et al. (2004b,a). The source was later detected by XMM-Newton (XMMU J005527.6-721059). The two X-ray positions obtained from Chandra and XMM-Newton are inconsistent with that of the proposed Be star counterpart. Furthermore, the X-ray spectrum is more typical of an AGN, making it highly likely that the period, detected with only $2.5 \sigma$ confidence, was spurious and the identification with the Be star was incorrect (Haberl \& Eger 2008).

XMMU J010016.1-720445 = RX J0100.2-7204 Additional XMM-Newton observations of source number 11 of Sasaki et al. (2003) provide an improved position that excludes identification with a bright optical counterpart. The X-ray source is classified as an AGN candidate (source 55 in Sturm et al. 2013c).

XMMU J010137.4-720418 = RXJ0101.6-7204 - This source is located close to the XMM-Newton calibration target 1E0102.2-7219 and was detected more than 30 times. The error-weighted X-ray position is incompatible with that of the emission-line star [MA93]1277 (Meyssonnier \& Azzopardi 1993), which was proposed by Sasaki et al. (2000) as possible counterpart in a BeXRB. The source appears as entry 21 in Shtykovskiy \& Gilfanov (2005). The steepness of the power-law $\mathrm{X}$-ray spectrum and the small X-ray variability of a factor of $\sim 4$ over $12 \mathrm{yr}$ of XMM-Newton observations are fully compatible with an AGN nature (source 53 in Sturm et al. 2013c).

AXJ0105-722 - The detection of a $3.34 \mathrm{~s}$ periodicity with $99.5 \%$ confidence $(2.8 \sigma)$ was claimed by Yokogawa \& Koyama (1998b). The X-ray spectrum was modelled with a power law with photon index $2.2 \pm 0.3$, which is much steeper than typically found for HMXBs in the $0.2-10 \mathrm{keV}$ band. RXJ0105.1-7211 was suggested as the most likely ROSAT counterpart of the ASCA source with MA93[1517] (Meyssonnier \& Azzopardi 1993) located 7.7" away from the
ROSAT position (Filipović et al. 2000a). Another nearby emission line star (MA93[1506]) was identified as Be star of spectral type B1-B2 III-Ve (with equivalent width of the $\mathrm{H} \alpha$ line of $-54 \AA$ ) and favoured as counterpart because it has an optical period of $11.09 \mathrm{~d}$ (Coe et al. 2005; McBride et al. 2008). However, such a short orbital period is not expected from the $\mathrm{H} \alpha$ - orbital period relation, which suggests on orbital period longer than $100 \mathrm{~d}$ (Fig. 9). Eger \& Haberl (2008a) used $X M M$-Newton observations to improve the X-ray position and classified XMMU J010509.7-721146 (power-law photon index $2.0 \pm 0.3$; no $V<18$ optical counterpart in error circle) as AGN. Positional coincidence and the agreement in X-ray spectral properties strongly suggest that AXJ0105-722, RX J0105.1-7211 and XMMU J010509.7-721146 are the same source and incompatible with [MA93] 1517 and [MA93] 1506 as optical counterpart. The $3.34 \mathrm{~s}$ periodicity is most likely spurious (but still appears in the list of Coe \& Kirk 2015).

XMMU J010519.9-724943 - As optical counterpart, Maravelias et al. (2014) suggested a B3-B5 star with $\mathrm{H} \alpha$ emission $4.0^{\prime \prime}$ from the X-ray position determined by these authors. Based on astrometric corrections and combining data from two XMM-Newton observations, Sturm et al. (2013c) derived a position 4.9" away from the optical position (source 420 in the $X M M-N e w t o n$ catalogue). This corresponds to $5 \sigma$, making the identification highly unlikely. Given the classification as AGN by Sturm et al. (2013c), we conclude that the Be star is not the counterpart of the X-ray source.

XMMU J010620.0-724049 - This case is very similar to the previous, here the separation Chandra/optical position is $4.5^{\prime \prime}$. Again XMM-Newton detections in two observations provide an X-ray position (source 426 in XMM-Newton SMC catalogue) that is even farther away (7.6"). A very likely AGN-counterpart is detected in Spitzer/IRAC images ${ }^{1}$ located only $0.64^{\prime \prime}$ from the $X M M$-Newton position. We conclude that the X-ray source is not a BeXRB, but an AGN.

\section{Confidence classes of HMXBs and candidates}

Candidates for HMXBs in the SMC were proposed in various publications mainly based on the positional coincidence of an X-ray source with an optical counterpart with appropriate brightness and colours consistent with an early-type star. The confidence with which they can be claimed to be a real HMXB or a BeXRB depends in particular on various aspects, such as the error of the X-ray position (usually significantly larger than the uncertainty in the optical position) and properties of the X-ray source as well as the candidate optical counterpart. This includes spectral and timing properties of the X-ray source and the optical star. When several criteria can be applied successfully, the confidence increases for a correct association of the X-ray source and the optical counterpart and its identification as an HMXB. Following this, we assigned flags to the (candidate) HMXBs in our compiled catalogue as described in Table 3.

Based on the flags, we divided the list of HMXBs and candidates into six confidence classes, which are summarised in Table 4. Sources with detected pulse period in X-rays (class I with flag ps or ps:) are most likely HMXBs even when the optical counterpart is not clearly identified as yet because the uncertainties in the X-ray position are large. X-ray sources with large long-term variability or a hard spectrum as typical for BeXRBs (in the $0.2-10 \mathrm{keV}$ band the photon index is lower than $\sim 1.3$;

1 SAGE LMC and SMC IRAC Source Catalog (IPAC 2009) available in Vizier. 
Table 3. Flags indicating different properties as measured for HMXBs and candidates.

\begin{tabular}{lll}
\hline \hline $\mathrm{C}^{a}$ & Flag $^{b}$ & Description \\
\hline 8 & ps & X-ray modulation indicates NS spin period \\
9 & px & long-term X-ray period suggests orbital period \\
10 & po & period in optical suggests orbital period \\
11 & os & orbital solution available \\
11 & ox & assuming X-ray period as orbital period \\
11 & oo & assuming optical period as orbital period \\
11 & oxo & X-ray and optical period are consistent \\
12 & xv & variability in X-rays larger than a factor 30 \\
13 & xs & typical X-ray spectrum \\
& & (power law with photon index $<1.3)$ \\
14 & oi & optical id with high confidence \\
15 & em & Balmer (H $\alpha$ ) emission measured from spectrum \\
15 & nem & no near-IR excess emission \\
\hline
\end{tabular}

Notes. ${ }^{(a)}$ Column in catalogue table (see Table 2). ${ }^{(b)}$ A colon behind the flag indicates an uncertain property.

Table 4. Summary of confidence classes.

\begin{tabular}{lll}
\hline \hline Class & Flags & Number of sources \\
\hline I & ps $\cup$ ps: & 63 \\
II & xv $\cup$ xs & 18 \\
III & oi $\cap$ em & 12 \\
IV & oi: $\cap$ em & 8 \\
V & oi & 3 \\
VI & oi: & 44 \\
\hline
\end{tabular}

Haberl et al. 2008) can also be identified as HMXBs from their $\mathrm{X}$-ray properties (class II with flag Xv or xs). A third class with secure identification of the optical counterpart (due to a precise $\mathrm{X}$-ray position) as an early-type emission-line star (flags oi and em) are also BeXRBs with high confidence. With increasing uncertainty in the X-ray position, the chance coincidence for the presence of an early-type star in the error circle increases. A larger X-ray position error is typically found for weak X-ray sources, which do not allow deriving other X-ray properties either. In class IV we list X-ray sources with less certain position and an emission-line star in the error circle (flags oi: and em). Class V comprises X-ray sources with a good position and therefore high confidence for their identification with early-type stars (flag oi). However, no information about $\mathrm{H} \alpha$ emission is available. Only three such sources are found in our catalogue, and optical spectra should easily allow confirming their probable Be-star counterpart. Finally, in class VI the highest fraction of chance correlations of the X-ray sources with early-type stars is expected. These are usually weak X-ray sources (flag oi: with position errors typically larger than $1^{\prime \prime}$ ), and apart from the appropriate colours, no other information about the possible optical counterpart is available.

As mentioned above, the probability for false identification of optical counterparts to the X-ray sources in class VI is higher than for classes I-V. The optical counterparts of the large majority of the BeXRB pulsars in class I are well investigated. Spectral types and the strength of the $\mathrm{H} \alpha$ emission were determined and can be found in the literature (e.g. Coe et al. 2005; McBride et al. 2008). Therefore, class I objects can be used to define a representative sample. Comparison of various source parameters between the different classes may then reveal differences, which indicate false identifications.
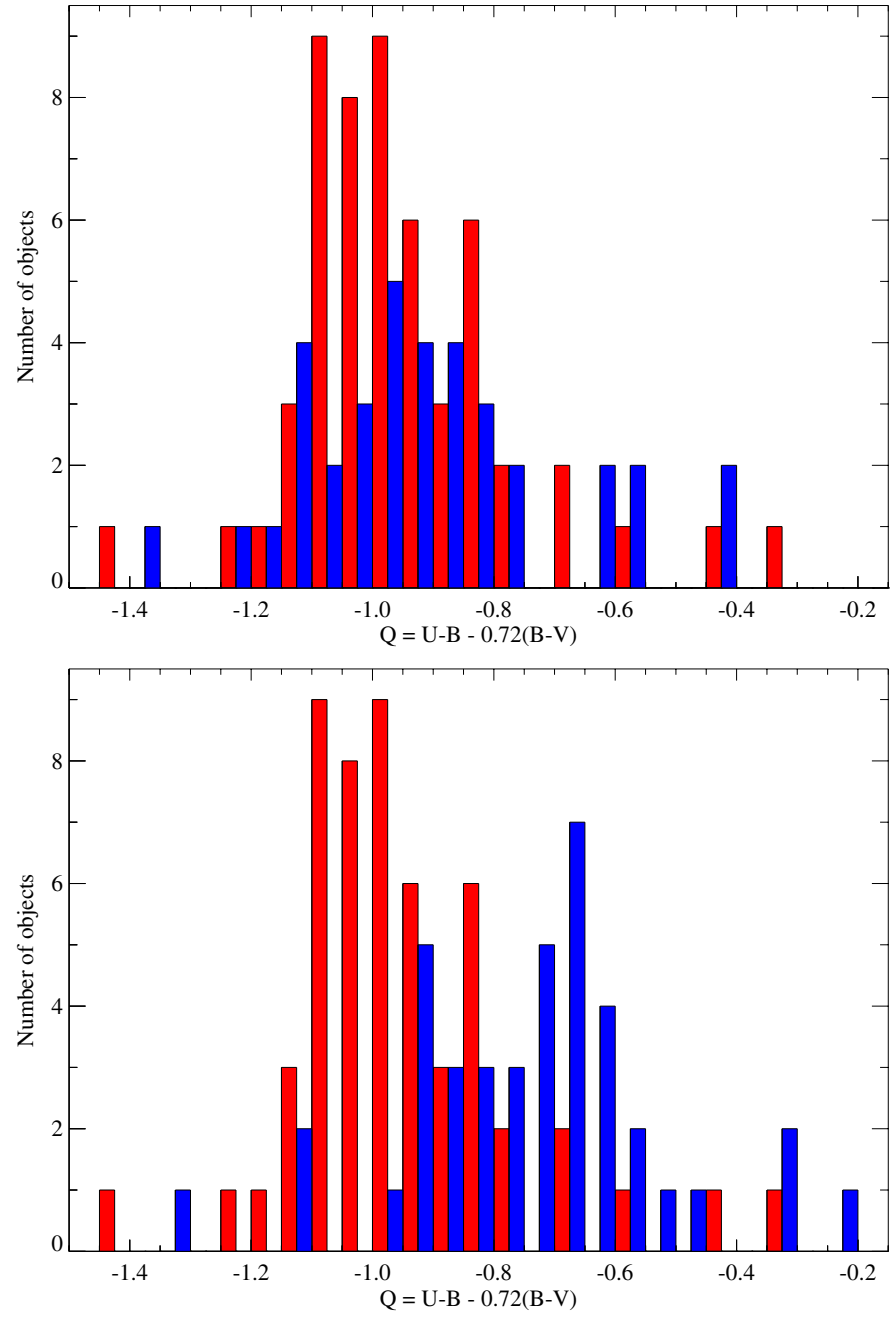

Fig. 3. Distribution of the reddening-free $Q$ parameter for different HMXB confidence classes. The top panel compares the pulsars (class I) with identified optical counterpart (red) with objects from classes II to V (blue), while in the bottom panel the comparison with class VI is shown.

When we plotted colour-colour $(U-B$ vs. $B-V)$ and colourmagnitude ( $V$ vs. $B-V$ ) diagrams, we found evidence that the objects in class VI are optically fainter and redder. To make this quantitatively clearer, we used the reddening-free $Q$-factor $Q=U-B-0.72(B-V)$ and compared its distribution between the different classes. While classes II-V are statistically consistent with class I, class VI exhibits a significantly different distribution towards higher $Q$ values. This is demonstrated in Fig. 3 where the distribution of the $Q$ parameter is compared for class I and class II-V (top panel) and for class I and class VI (bottom). A statistical Kolmogorov-Smirnov test results in a probability of $98.5 \%$ for class I and II-V and $1.3 \times 10^{-7}$ for class I and VI to be drawn from the same distribution. This clearly shows that the early-type stars proposed as optical counterparts of the X-ray sources from class VI are on average of different (later) spectral type than the optical counterparts of class I, which show a relatively narrow distribution around B0-B1 (McBride et al. 2008). In particular, no spectral type later than $B 2$ was found for BeXRBs with well-known optical counterpart in the Milky Way (Reig 2011). On the other hand, isolated Be stars with later spectral type exist (McBride et al. 2008), and we cannot exclude that (in particular X-ray faint) class VI objects might be associated with a late-type BeXRB. However, the larger X-ray error 


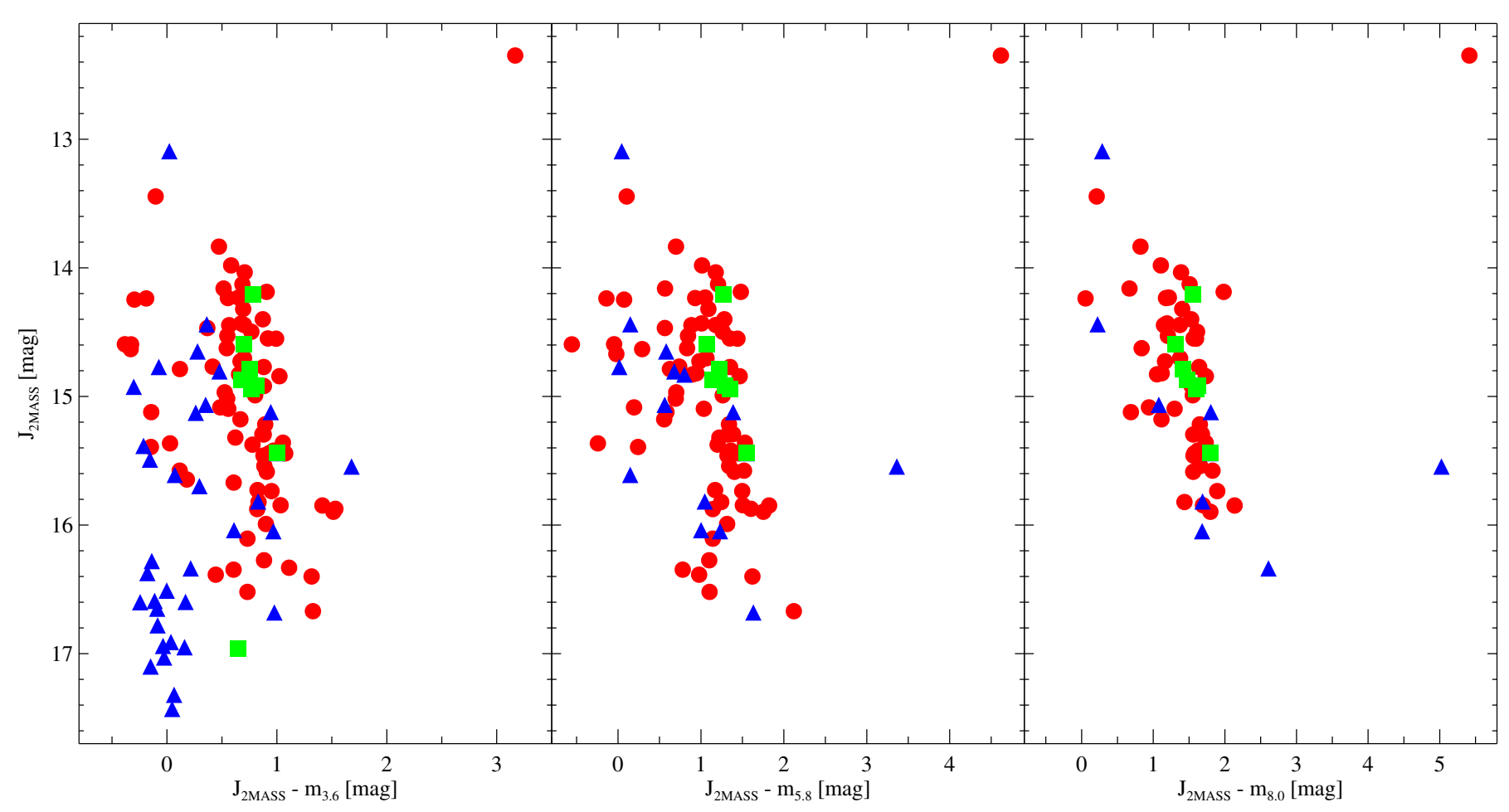

Fig. 4. Infrared excess using the three IRAC magnitudes at 3.6, 5.8, and $8.0 \mu \mathrm{m}$ and $J$ band from $2 \mathrm{MASS}$ (in a few cases from IRSF when no 2MASS value was available). Blue triangles mark sources from class VI (flag "oi:"), green squares those from class IV (with additional flag "em"), while red circles indicate flag "oi".

circles of the faint class VI objects implies that many of them are chance coincidences of the X-ray sources with single late B- or even Be-type stars.

Bonanos et al. (2010) have used Spitzer IRAC fluxes (at 3.6, $4.5,5.8$, and $8.0 \mu \mathrm{m}$ ) of $\mathrm{O}$ and early-B stars in the SMC to distinguish $\mathrm{OBe}$ from $\mathrm{OB}$ stars based on their infrared excess relative to $J$-band fluxes. In Fig. 4 we plot the infrared excess for the catalogue sources with flags "oi" and "oi:". A remarkably large portion of sources from class VI (16 out of 44) fall in a region with $J>16$ and $J-m_{3.6}<0.3$ where no other sources are found. This suggests that these 16 objects are most likely normal B stars without a circum-stellar disc (Bonanos et al. 2010, where a threshold of $J-m_{3.6}>0.5$ is defined to introduce a photometric Be star classification). Fourteen of the sixteen objects are from the BeXRB candidate list derived from the XMM-Newton survey of the SMC (sources 41, 259, 468, 474, 1019, 1189, 1762, 1955, 2211, 2569, 2675, 2721, and 2967, 3271 from Sturm et al. 2013c). The authors determined the number of chance coincidences as high as twenty. Therefore, the lack of infrared excess excludes the greater part of the expected chance coincidences. The remaining two objects (CXO J005331.8-721845 and CXO J005419.2-722049) are from the candidate list of Laycock et al. (2010), very faint X-ray sources with positional errors larger than $5^{\prime \prime}$. Neither are found in the CSC. The proposed optical counterparts have optical colours consistent with an earlytype star. They do not show infrared excess, which suggests that they are not Be stars and therefore are most unlikely the counterparts of the weak Chandra sources, which might even be spurious detections after all. This is also supported by their Q-values, which are $>-0.91$. We flag these sixteen objects with "nem" in the catalogue.

As expected, the class IV objects are among the wellestablished BeXRBs, as is shown by their flag "em". However, it should be noted that for class IV objects a misidentification of the X-ray source with a Be star can still not be excluded: as a result of their larger X-ray position uncertainty, the number of chance coincidences is higher than for objects whose optical counterpart is unambiguously identified (flag "oi").

\section{Candidates that are probably misidentified as HMXBs}

In Sect. 3 we have shown that a large portion of the 44 sources in confidence class VI is most likely misidentified as HMXBs. New data for some of the proposed candidates further contradict their proposed HMXB nature. In the following we list objects that we finally consider to probably not be HMXBs or which we reject.

Sturm et al. (2013c) present a list of 45 HMXB candidates selected from their SMC X-ray source catalogue (see their Table 5). Taking into account the positional uncertainties of the $\mathrm{X}$-ray sources, they estimate $16.6 \pm 3.4$ chance coincidences. Meanwhile, additional information is available for many of the candidates, which allows us to better constrain the origin of their $\mathrm{X}$-ray emission. This includes Chandra X-ray data (with source positions often available from the on-line catalogue of Evans et al. 2010), possible AGN counterparts properly selected from Spitzer data using colour indices (SAGE LMC and SMC IRAC Source Catalog - IPAC 2009), and absence of a near-IR excess, normally seen from Be stars (see Sect. 3). In particular, McBride et al. (2016) have obtained optical spectroscopy of all candidates presented by Sturm et al. (2013c), and in several cases no Balmer emission was found (although $\mathrm{H} \alpha$ was not always covered). It should be noted here that Be stars can lose and rebuild their circum-stellar disc, as is indicated by the disc emission coming and going on timescales of decades (Rivinius et al. 2013) with 
state transitions as short as a few months (e.g. Reig et al. 2007). Although we consider a disc loss between X-ray and optical observations unlikely, we cannot exclude that a BeXRB is observed during a disc-less state. Therefore, we label cases without an indication of a circum-stellar disc because there is no near-IR excess or Balmer emission as "HMXB unlikely" and only cases without near-IR excess and no Balmer emission as "HMXB rejected" in the comment column of the catalogue. We kept the unlikely/rejected cases in our catalogue. We also rejected candidates with improved X-ray positions that exclude the previously suggested counterparts, candidates that are more probably AGN because of their Spitzer counterparts and other information (summarised below).

At the current status of our work, this means that we have 27 candidates in total (11 marked as unlikely and 16 as rejected) that probably are HMXB misidentifications. Twenty-five of them belong to confidence class VI, only one was given class III ([SHP2013] 1408, see below) and one class V ([SHP2013]287, see below). Twenty of the twenty-seven sources are from the candidate list of Sturm et al. (2013c). From this list we can confirm fourteen sources as HMXBs, and for eleven cases no additional information is available and they remain candidates. Following SIMBAD naming conventions, we use [SHP2013]N for source number $N$ in the catalogue of Sturm et al. (2013c) and [SG2005] SMC N for sources in Table 2 of Shtykovskiy \& Gilfanov (2005).

For [SHP2013] $117=[$ SG2005] SMC 34 a $V=16.2 \mathrm{mag}$ star was proposed as the optical counterpart. Sturm et al. (2013a) argued for QSO J004818.72-732059.83 (Kozłowski \& Kochanek 2009) as another likely counterpart, very close to the X-ray position. McBride et al. (2016) found no Balmer emission from the early-type star, making the QSO the more likely counterpart.

A $V=16.7$ mag star (with spectral type B2 V Nazé et al. 2003b) was proposed as counterpart for [SHP2013] $160=$ [SG2005] SMC 39. No Balmer emission McBride et al. (2016) and the presence of a Spitzer counterpart make an AGN nature more likely.

[SHP2013]259 $=$ [SG2005] SMC 48 was identified as an eclipsing binary with an orbital period of 5.18 days (Wyrzykowski et al. 2004). This makes an HMXB nature highly unlikely.

A $V=15.1$ mag star (with spectral type B1-3 III from the $2 \mathrm{dF}$ survey of the SMC, Evans et al. 2004) was proposed as the counterpart for [SHP2013] 287. McBride et al. (2016) found no Balmer emission from the early-type star, suggesting a chance coincidence with the X-ray source.

The proposed counterpart of [SHP2013] 474 shows no nearIR excess (see Sect. 3), and McBride et al. (2016) found no Balmer emission.

For [SHP2013]562 a $V=15.4$ mag star with spectral type B0 V from the $2 \mathrm{dF}$ survey (Evans et al. 2004) was proposed as counterpart. McBride et al. (2016) found no Balmer emission.

The angular distance between [SHP2013] 1019 and its proposed optical counterpart is 3.5". McBride et al. (2016) found no Balmer emission. No near-IR excess is seen either (see Sect. 3). The presence of a Spitzer counterpart makes a chance coincidence with the early-type star most likely and suggests an AGN origin for the X-ray source.

[SHP2013] 1189 is located about 3.5' from 1E0102.2-7219, the X-ray brightest supernova remnant in the SMC that is frequently observed by XMM-Newton. The faint source was detected only once with a large positional error, and no entry is found in the Chandra on-line catalogue near its position.
However, the source is clearly seen in the merged Chandra ACIS-I image presented in Sturm et al. (2013c), which suggests a constantly faint source. The Chandra position is incompatible with that of the $V=16.1 \mathrm{mag}$ star proposed as counterpart. This star also shows no near-IR excess.

[SHP2013] 1408 is associated with the B[e] super-giant star LHA 115-S 18 (Clark et al. 2013; Maravelias et al. 2014), and the X-ray emission is probably not produced by accretion onto a compact object.

The case of [SHP2013]1762 is very similar to [SHP2013] 1189: near 1E0102.2-7219; seen in the merged Chandra ACIS-I image; Chandra position incompatible with the $V=16.3$ mag star proposed as counterpart, which shows no near-IR excess.

McBride et al. (2016) identified the proposed counterpart to [SHP2013] 1823 as Be star. However, the uncertain XMM-Newton position is at a distance of $4.9^{\prime \prime}$. The source was also detected in Chandra data (Laycock et al. 2010), also 5" from the optical position. The Chandra $95 \%$ uncertainty of $1.83^{\prime \prime}$ makes the identification of the X-ray source with the Be highly unlikely. Moreover, the existence of a Spitzer counterpart suggests an AGN origin.

The XMM-Newton detection of the weak source [SHP2013] 1826 yields a highly uncertain position and is $5.7^{\prime \prime}$ away from its proposed optical counterpart. In addition, a Chandra detection $\left(95 \%\right.$ error $=0.47^{\prime \prime}$, distance 5.4" Laycock et al. 2010) excludes an identification with the proposed $V=14.9$ mag star.

The proposed counterpart of [SHP2013] 1955 shows no near-IR excess (Sect. 3), and McBride et al. (2016) found no Balmer emission. We conclude that the X-ray source is not associated with the normal B star.

The angular distance between [SHP2013] 2100 and its proposed optical counterpart is $4.3^{\prime \prime}$, and McBride et al. (2016) found no Balmer emission.

The position of the proposed counterpart of [SHP2013] 2318 is 5.7" away. We determined an improved position from a recent deep Chandra observation (ID 14671) to an accuracy of $0.3^{\prime \prime}(1 \sigma)$. The Chandra position is within 2.0" of the $X M M$-Newton position, but with 6.9" distance incompatible with that of the $V=16.1$ mag early-type star.

McBride et al. (2016) found no Balmer emission from the suggested counterpart of [SHP2013]2497, which makes a chance correlation with a normal B star most likely.

No near-IR excess and no Balmer emission are seen from the suggested counterpart of [SHP2013] 2569 McBride et al. (2016). The existence of a Spitzer counterpart suggests an AGN origin.

[SHP2013] 2675 is another case that was identified as an eclipsing binary (orbital period of 3.29 days Wyrzykowski et al. 2004), which makes an HMXB nature highly unlikely. No nearIR excess is seen from the binary star system.

For [SHP2013] 2737 a $V=14.7$ mag star with spectral type B5 II from the 2dF survey (Evans et al. 2004) was proposed as counterpart. McBride et al. (2016) found no Balmer emission. A Chandra detection (Evans et al. 2010) yields a position 2.9" from the B star. A Spitzer counterpart suggests an AGN origin.

The $V=16.6 \mathrm{mag}$ star suggested as counterpart for [SHP2013] 3271 is 4.4" away from the XMM-Newton position. McBride et al. (2016) found no $\mathrm{H} \alpha$ emission. No detected nearIR excess is consistent with a normal B star found by chance in the X-ray error circle. 


\section{Population statistics}

The large number of HMXBs in the SMC allows statistical investigations of their X-ray and optical properties. Many BeXRBs were found because coherent pulsations in the X-ray flux (often during outburst) were detected, which indicates the spin period of the NS. Knigge et al. (2011) discussed the bimodal distribution of the spin period with two maxima at around $10 \mathrm{~s}$ and between $100 \mathrm{~s}$ to $1000 \mathrm{~s}$ in terms of two populations of $\mathrm{X}$-ray pulsars produced by two types of supernovae. As an alternative explanation, Cheng et al. (2014) proposed that the spin period distribution is the result of two different accretion modes. To elaborate on this question, we collected maximum and minimum fluxes (or upper limits when lower) reported for the SMC BeXRBs in the literature and entered them in our catalogue. The high sensitivity of the XMM-Newton SMC survey provides stringent upper limits for non-detected sources. These are available in the catalogue of Sturm et al. (2013c) for sources observed more than once and detected at least once. For the remaining SMC HMXBs, which were never detected in any XMM-Newton observation, we readout the upper limits from the sensitivity maps produced for the work of Sturm et al. (2013c). If an XMM-Newton upper limit was higher than any detected (minimum) flux from another instrument, then it was not used. In Fig. 5 we plot the maximum and minimum fluxes $\left(F_{\max }\right.$ and $\left.F_{\min }\right)$ and the derived ratio (variability factor) for the SMC BeXRB pulsars versus their spin period. As shown by Cheng et al. (2014), short-period (<40 s) pulsars show on average higher maximum luminosities than long-period pulsars. This is demonstrated by the rather loose anti-correlation of maximum flux with spin period in the upper panel of Fig. 5. We note that the minimum observed flux (or upper limit for non-detections) also tends to be lower for the short-period pulsars. While most of the short-period pulsars even fall below the detection limits of modern X-ray instrumentation, many long-period pulsars are always detected well above these limits (Fig. 5, middle panel). As a consequence, the anti-correlation of $F_{\max } / F_{\text {min }}$ with spin period is even more pronounced (Fig. 5, lower panel). There still might be some observational bias in the correlations, since some systems have not been caught at outburst maximum or at their minimum flux level and the observed $F_{\max } / F_{\min }$ value presents only a lower limit. However, given the large number of X-ray observations, this should affect only a relatively small number of objects independent of spin period and would not change the overall distributions. It is therefore remarkable that the upper envelope of the data points in the bottom panel of Fig. 5 is so sharp, suggesting that the neutron stars with longer spin periods - and wider orbits (Fig. 8) - sample a narrower range in accretion rate than short-period pulsars. The largest variations in accretion rate are expected for systems with high eccentricity and/or large tilt between orbital plane and Be disc.

For six of SMC XRBs the eccentricity of the orbit is known with values between 0.07 and 0.43 . Interestingly, among these, SMC X-2 has the lowest eccentricity and the highest variability factor of $\sim 5 \times 10^{4}$. Although the orbital parameters could be determined only for neutron stars with short spin periods (which show the highest variability), the measured eccentricities are relatively moderate. This may indicate that eccentricity is not the main parameter leading to the strong outbursts from these systems. Alternatively, a considerable inclination of the orbit with respect to the plane of the circum-stellar disk can also lead to strong and in particular short outbursts when the NS passes through the disc. Strong evidence for this kind of system geometry was found for SXP5.05 (Coe et al. 2015). A high

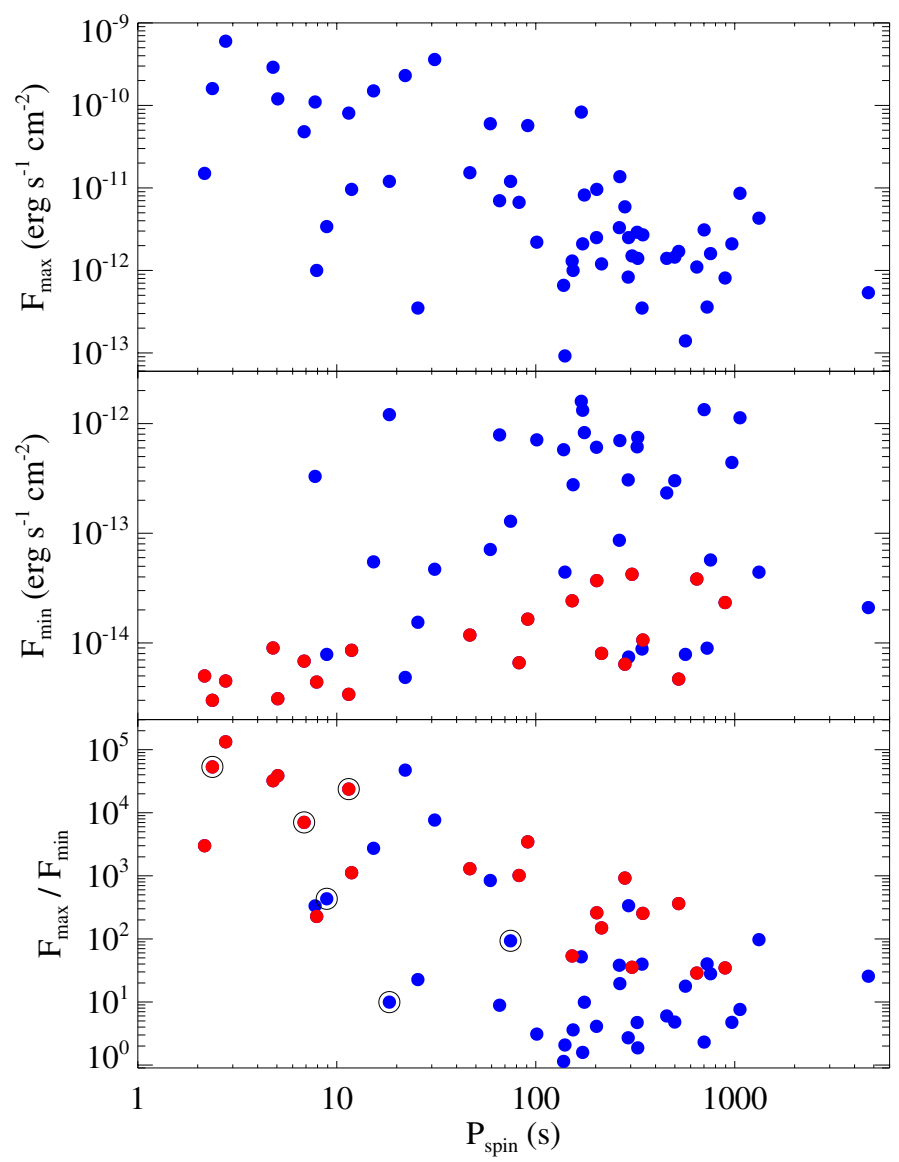

Fig. 5. Observed maximum (top) and minimum (middle) X-ray flux together with their ratio (bottom) as function of spin period. When sources were not detected in a second observation, available upper limits were used for the minimum flux (marked in red). Black circles in the bottom panel indicate the BeXRBs with measured orbit eccentricity.

inclination with the NS moving far above and below the disc can also explain the lower minimum X-ray flux values observed from systems with short spin period (and high variability). Moreover, such a system geometry is thought to cause a strong warping of the disc, which then can lead to the strong X-ray outbursts and high spin-up of the NS to short periods (Cheng et al. 2014).

While maximum fluxes seen from BeXRBs are mainly determined by the available supply of matter along the neutron star orbit, the observed minimum flux is very likely influenced by another mechanism. The propeller effect can inhibit accretion when the matter from the accretion disc couples onto the rotating magnetosphere of the neutron star at distances larger than the co-rotation radius (Stella et al. 1986). The critical luminosity strongly depends on the spin period (Eq. (5) of that paper) and is expected to be about $2.7 \times 10^{36} \mathrm{erg} \mathrm{s}^{-1}$ for instance for SMCX-2 with a period of $2.37 \mathrm{~s}$. The large variability factors of short-period pulsars might be explained by this mechanism. When magnetospheric accretion stops, only faint emission from the (cooling) neutron star surface remains, possibly with some small contribution from direct accretion. Several BeXRB pulsars observed in extreme low states at X-ray luminosities of $\sim 10^{34} \mathrm{erg} \mathrm{s}^{-1}$ and below are believed to be seen in the propeller state (see Raguzova \& Popov 2005, and references therein). For long-period pulsars the propeller effect is thought to play no role (e.g. for a period of $400 \mathrm{~s}$ the critical luminosity is at $\left.1.7 \times 10^{31} \mathrm{erg} \mathrm{s}^{-1}\right)$. However, we note 


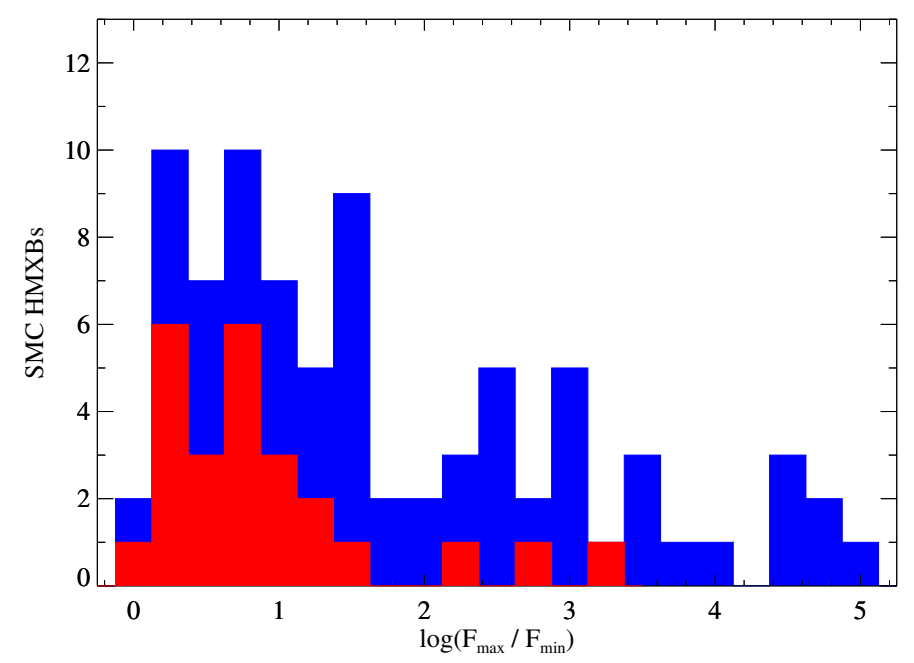

Fig. 6. Distribution of the variability factor $F_{\max } / F_{\min }$ for all HMXBs with confidence class I-V (blue) and for the non-pulsars (confidence class II-V, red) in the SMC.

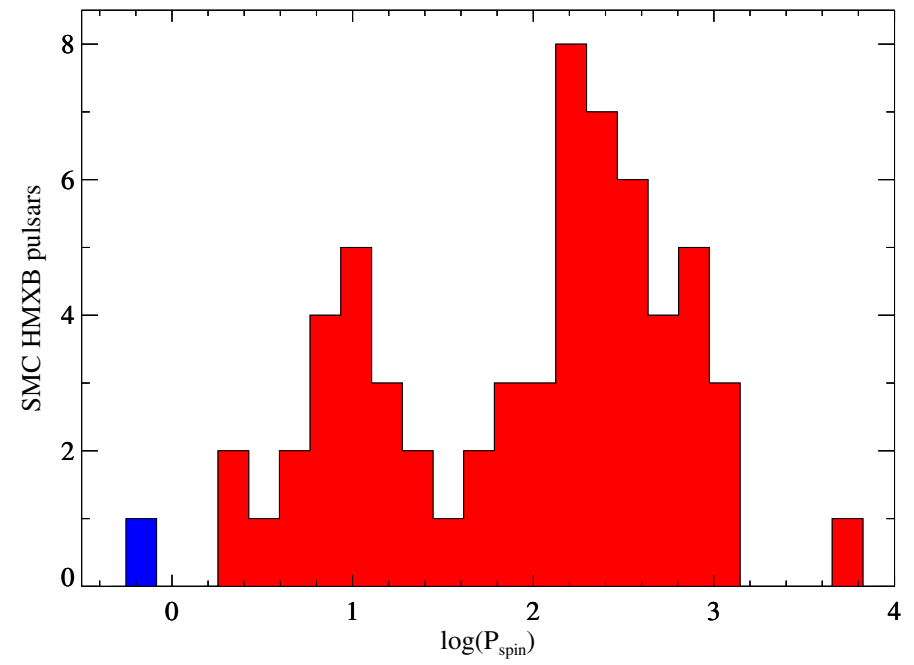

Fig. 7. Spin period histogram for $63 \mathrm{HMXB}$ pulsars in the SMC. BeXRBs are shown in red, while the super-giant system SMCX-1 is depicted in blue.

here that Eger \& Haberl (2008a) reported a sharp drop in X-ray luminosity below the detection limit of XMM-Newton from SAX J0103.2-7209. The drop occurred after the source reached a level of constant spin period (after years of spin-up) at a luminosity of about $3 \times 10^{35} \mathrm{erg} \mathrm{s}^{-1}$ and a small further decrease to $2 \times 10^{35} \mathrm{erg} \mathrm{s}^{-1}$. The authors suggest that this behaviour could be qualitatively explained by the propeller effect, but at a critical luminosity four orders of magnitude higher than expected from Eq. (5) of Stella et al. (1986).

Figure 6 demonstrates that the distributions of the variability factor among BeXRB pulsars and sources without detected pulsations in confidence classes II-V are also similar. If the anti-correlation of variability and spin period also holds for the sources that have no detected spin period (yet), this suggests that most class II-V sources probably have longer spin periods, which would contribute to the long-period peak in the spin-period distribution of Fig. 7. Because sufficiently long observations are required to detect these periods, this could be one reason why they have not yet been found. Conversely, only three (one) class II-V objects have variability factors greater than

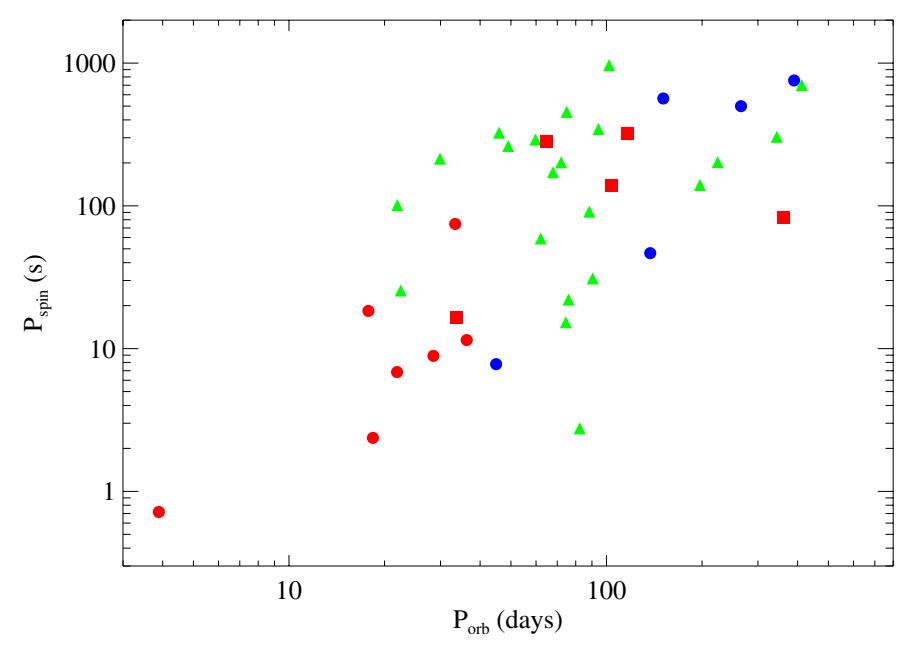

Fig. 8. Spin period vs. orbital period for HMXBs in the SMC. The super-giant HMXB SMC X-1 is found in the lower-left corner. Orbital periods found with different methods are marked with different symbols: orbit solution (red circles), X-ray outbursts (red squares), optical light curve (green triangles). Blue circles mark systems with orbital periods consistently derived from $\mathrm{X}$-rays and optical.

100 (1000). For all three, few X-ray observations exist in general, and none have sufficient statistics to allow a sensitive period search.

In Fig. 7 we present the updated spin period histogram for SMC pulsars, which also includes the two objects with lowsignificance detections of the period, which need to be confirmed: the $6.878 \mathrm{~s}$ detected in Integral data (McBride et al. 2007) and the 154s period from XMMU J010743.1-715953 (Coe et al. 2012). We also include the long 4693 s period found by Laycock et al. (2010) from a $100 \mathrm{ks}$ Chandra observation that may be present in a $23 \mathrm{ks} X M M-N e w t o n$ observation (see Sect. 2.2).

Orbital periods of HMXBs and in particular from BeXRBs can be identified from their long-term light curves in X-rays and optical. Long-term monitoring of the SMC pulsars in X-rays was performed by RXTE (Galache et al. 2008), which was sensitive to periodic outbursts near periastron passage of the NS. The OGLE project has monitored stars in the SMC for more than a decade and revealed periodic long-term variability for many Be stars in BeXRBs, which can be attributed to the orbital period of the binary system (Rajoelimanana et al. 2011b). For a few systems the Doppler analysis of the X-ray pulse timing data allowed deriving orbital solutions (e.g. Townsend et al. 2011a). In Fig. 8 we show the spin versus orbital period diagram for the HMXBs in the SMC methodised by different symbols. The first version of such a diagram (Corbet 1984) showed a strong correlation between the two parameters for BeXRBs. Our most recent version contains five times more systems and the correlation has considerably weakened. The correlation is thought to be explained by a quasi-equilibrium state of the neutron star in which the co-rotation radius equals the Alfvén radius. In this picture, the rotation period and the magnetic field strength of the neutron star and the accretion rate determine whether matter can be accreted onto the neutron star (and spin it up) or not (leading to spin-down). The large scatter seen in our updated diagram (the spread in spin period for orbital periods between 50 and 100 days is $\sim 2.5$ orders of magnitude) suggests that many of the neutron stars do not rotate near their equilibrium rate, even bearing in mind that the model is too simple. 
F. Haberl and R. Sturm: High-mass X-ray binaries in the SMC

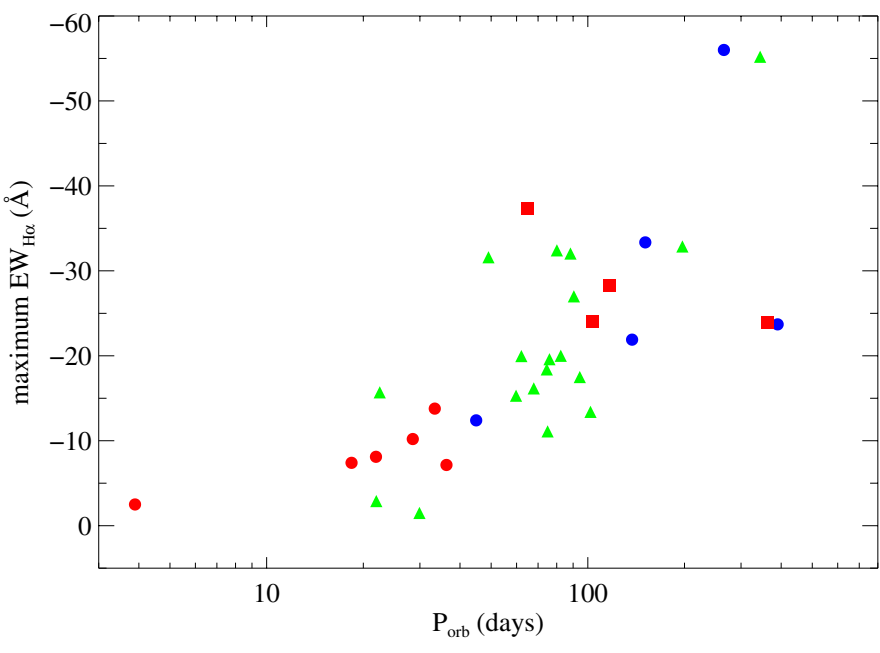

Fig. 9. Equivalent width of the $\mathrm{H} \alpha$ line vs. orbital period for HMXBs in the SMC. The symbols mark the different origin of orbital periods as in Fig. 8. When more than one measurement of the equivalent width is available the largest (most negative) value is used.

In Fig. 9 we present another diagram that is important for characterising the properties of BeXRBs; the equivalent width (observed maximum) is plotted as function of orbital period. For all except one of the objects in this figure, that is, all whose orbital period is known and that have $\mathrm{H} \alpha$ measurements, the spin period is known as well. The exception is the unclear case of RX J0049.2-7311, which we do not associate with SXP9.13 (see Sect. 2.1.1). If the minimum equivalent width is used for objects with more than one $\mathrm{H} \alpha$ measurement, the plot is not significantly altered. Coe \& Kirk (2015) investigated the dependence of the equivalent width on orbital period in more detail, in particular with respect to the size of the circum-stellar disc and disc truncation by the compact object (see also Reig et al. 1997). Since in their work SXP9.13 is identified with RX J0049.2-7311, our whole sample from Fig. 9 is covered in their analysis, and we refer to that work for more details.

\section{Conclusions}

We investigated the properties of 148 (candidate) HMXBs in the SMC and catalogued them. We assigned different levels of confidence at which they are genuine HMXB systems for all catalogue entries. Pulsars are confidence class I, and the probability for being HMXBs decreases until class VI objects. We found many chance coincidences between X-ray and optical position in class VI and rejected 27 candidates as probably misidentified with normal B stars.

The remaining 121 sources comprise a relatively clean sample of HMXBs in the SMC. With 63 pulsars, this indicates that almost $50 \%$ of the sources do not have a detected pulse period. A comparison of X-ray variability as function of spin period for pulsars and "non-pulsars" suggests that many long spin periods (longer than a few $100 \mathrm{~s}$ ) have probably not been found as yet because of the intrinsic short-term X-ray variability of BeXRBs and insufficient observation time. Therefore, it remains unclear how many neutron stars in BeXRBs do not exhibit pulsations because their magnetic field axis is (nearly) aligned with the rotation axis. The larger long-term X-ray variability of objects with short spin period also indicates different accretion schemes for short and long spin period pulsars, which may favour the accretion model of Cheng et al. (2014) to explain the bimodal distribution of spin periods observed from BeXRBs.
Acknowledgements. R.S. acknowledges support from the BMWI/DLR grant FKZ 50 OR 0907 during his stay at MPE.

Note added in proof. A recent observation with XMM-Newton has shown that catalogue entries number 2 and 142 belong to the same BeXRB (Haberl et al. 2015).

\section{References}

Antoniou, V., Hatzidimitriou, D., Zezas, A., \& Reig, P. 2009a, ApJ, 707, 1080 Antoniou, V., Zezas, A., Hatzidimitriou, D., \& McDowell, J. C. 2009b, ApJ, 697, 1695

Azzopardi, M., Vigneau, J., \& Macquet, M. 1975, A\&AS, 22, 285

Bonanos, A. Z., Lennon, D. J., Köhlinger, F., et al. 2010, AJ, 140, 416

Buccheri, R., Bennett, K., Bignami, G. F., et al. 1983, A\&A, 128, 245

Buckley, D. A. H., Coe, M. J., Stevens, J. B., et al. 2001, MNRAS, 320, 281

Chakrabarty, D., Levine, A. M., Clark, G. W., \& Takeshima, T. 1998a, IAU Circ., 7048, 1

Chakrabarty, D., Takeshima, T., Ozaki, M., Paul, B., \& Yokogawa, J. 1998b, IAU Circ., 7062, 1

Cheng, Z.-Q., Shao, Y., \& Li, X.-D. 2014, ApJ, 786, 128

Clark, G. W., Remillard, R. A., \& Woo, J. W. 1997, ApJ, 474, L111

Clark, J. S., Bartlett, E. S., Coe, M. J., et al. 2013, A\&A, 560, A10

Coe, M. J., \& Kirk, J. 2015, MNRAS, 452, 969

Coe, M. J., \& Orosz, J. A. 2000, MNRAS, 311, 169

Coe, M. J., Haigh, N. J., Laycock, S. G. T., Negueruela, I., \& Kaiser, C. R. 2002, MNRAS, 332, 473

Coe, M. J., Edge, W. R. T., Galache, J. L., \& McBride, V. A. 2005, MNRAS, 356,502

Coe, M. J., Schurch, M., McBride, V. A., et al. 2009, MNRAS, 394, 2191

Coe, M. J., Bird, A. J., Buckley, D. A. H., et al. 2010, MNRAS, 406, 2533

Coe, M. J., Bartlett, E. S., Bird, A. J., et al. 2011a, ATel, 3396, 1

Coe, M. J., Haberl, F., Sturm, R., et al. 2011b, MNRAS, 414, 3281

Coe, M. J., Haberl, F., Sturm, R., et al. 2012, MNRAS, 424, 282

Coe, M. J., Bird, A. J., Kennea, J. A., et al. 2013a, ATel, 5662, 1

Coe, M. J., Bird, A. J., McBride, V., Bartlett, E. S., \& Haberl, F. 2013b, ATel, 5547,1

Coe, M. J., Bird, A. J., McBride, V. A., et al. 2014, ATel, 5806, 1

Coe, M. J., Bartlett, E. S., Bird, A. J., et al. 2015, MNRAS, 447, 2387

Corbet, R. H. D. 1984, A\&A, 141, 91

Corbet, R., Marshall, F. E., Lochner, J. C., Ozaki, M., \& Ueda, Y. 1998, IAU Circ., 6803, 1

Corbet, R., Markwardt, C. B., Marshall, F. E., Laycock, S., \& Coe, M. 2002, IAU Circ., 7932, 2

Corbet, R. H. D., Markwardt, C. B., Marshall, F. E., et al. 2003, ATel, 163, 1

Corbet, R. H. D., Coe, M. J., Marshall, F. E., McBride, V. A., \& Schurch, M. P. E. 2008, ATel, 1600, 1

Corbet, R. H. D., Bartlett, E. S., Coe, M. J., et al. 2010, ATel, 2813, 1

Covino, S., Negueruela, I., Campana, S., et al. 2001, A\&A, 374, 1009

Cowley, A. P., Schmidtke, P. C., McGrath, T. K., et al. 1997, PASP, 109, 21

Eger, P., \& Haberl, F. 2008a, A\&A, 491, 841

Eger, P., \& Haberl, F. 2008b, A\&A, 485, 807

Edge, W. R. T., \& Coe, M. J. 2003, MNRAS, 338, 428

Edge, W. R. T., Coe, M. J., Corbet, R. H. D., et al. 2003, ATel, 215, 1

Edge, W. R. T., Coe, M. J., Galache, J. L., et al. 2004a, MNRAS, 353, 1286

Edge, W. R. T., Coe, M. J., \& McBride, V. A. 2004b, ATel, 217, 1

Esposito, P., Israel, G. L., Sidoli, L., et al. 2013, MNRAS, 433, 3464

Evans, C. J., Howarth, I. D., Irwin, M. J., Burnley, A. W., \& Harries, T. J. 2004 MNRAS, 353, 601

Evans, C. J., Lennon, D. J., \& Smartt, S. J. 2006, in Stellar Evolution at Low Metallicity: Mass Loss, Explosions, Cosmology, eds. H. J. G. L. M. Lamers, N. Langer, T. Nugis, \& K. Annuk, ASP Conf. Ser., 353, 41

Evans, I. N., Primini, F. A., Glotfelty, K. J., et al. 2010, ApJS, 189, 37

Filipović, M. D., Haberl, F., Pietsch, W., \& Morgan, D. H. 2000a, A\&A, 353, 129

Filipović, M. D., Pietsch, W., \& Haberl, F. 2000b, A\&A, 361, 823

Galache, J. L., Corbet, R. H. D., Coe, M. J., et al. 2008, ApJS, 177, 189

Gregory, P. C., \& Loredo, T. J. 1996, ApJ, 473, 1059

Haberl, F., \& Eger, P. 2008, ATel, 1529, 1

Haberl, F., \& Pietsch, W. 2004, A\&A, 414, 667

Haberl, F., \& Pietsch, W. 2005, A\&A, 438, 211

Haberl, F., \& Sasaki, M. 2000, A\&A, 359, 573

Haberl, F., Filipović, M. D., Pietsch, W., \& Kahabka, P. 2000, A\&AS, 142, 41

Haberl, F., Pietsch, W., Schartel, N., Rodriguez, P., \& Corbet, R. H. D. 2004, A\&A, 420, L19 
Haberl, F., Pietsch, W., \& Kahabka, P. 2007, ATel, 1095, 1 Haberl, F., Eger, P., \& Pietsch, W. 2008, A\&A, 489, 327

Haberl, F., Sturm, R., Filipović, M. D., Pietsch, W., \& Crawford, E. J. 2012a, A\&A, 537, L1

Haberl, F., Sturm, R., Tsujimoto, M., et al. 2012b, ATel, 4648, 1

Haberl, F., Vasilopoulos, G., Sturm, R., \& Maggi, P. 2015, ATel, 8305, 1

Hénault-Brunet, V., Oskinova, L. M., Guerrero, M. A., et al. 2012, MNRAS, 420, L13

Hughes, J. P., \& Smith, R. C. 1994, AJ, 107, 1363

Imanishi, K., Yokogawa, J., \& Koyama, K. 1998, IAU Circ., 7040, 2

Israel, G. L., Stella, L., Angelini, L., et al. 1997, ApJ, 484, L141

Israel, G. L., Stella, L., Campana, S., et al. 1998, IAU Circ., 6999, 1

Israel, G. L., Stella, L., Covino, S., Campana, S., \& Mereghetti, S. 1999, IAU Circ., 7101, 1

Israel, G. L., Campana, S., Covino, S., et al. 2000, ApJ, 531, L131

Israel, G. L., Esposito, P., D’Elia, V., \& Sidoli, L. 2013, ATel, 5552, 1

Kahabka, P., \& Hilker, M. 2005, A\&A, 435, 9

Kahabka, P., \& Pietsch, W. 1996, A\&A, 312, 919

Kahabka, P., Pietsch, W., Filipović , M. D., \& Haberl, F. 1999, A\&AS, 136, 81

Keller, S. C., Wood, P. R., \& Bessell, M. S. 1999, A\&AS, 134, 489

Kennea, J. A. 2011, ATel, 3578, 1

Knigge, C., Coe, M. J., \& Podsiadlowski, P. 2011, Nature, 479, 372

Kourniotis, M., Bonanos, A. Z., Soszyński, I., et al. 2014, A\&A, 562, A125

Kozłowski, S., \& Kochanek, C. S. 2009, ApJ, 701, 508

Lamb, R. C., Prince, T. A., Macomb, D. J., \& Finger, M. H. 1999, IAU Circ., 7081, 4

Lamb, R. C., Macomb, D. J., Prince, T. A., \& Majid, W. A. 2002, ApJ, 567, L129

Laycock, S., Corbet, R. H. D., Perrodin, D., et al. 2002, A\&A, 385, 464

Laycock, S., Corbet, R. H. D., Coe, M. J., et al. 2005, ApJS, 161, 96

Laycock, S., Zezas, A., Hong, J., Drake, J. J., \& Antoniou, V. 2010, ApJ, 716, 1217

Liu, Q. Z., van Paradijs, J., \& van den Heuvel, E. P. J. 2005, A\&A, 442, 1135

Macomb, D. J., Finger, M. H., Harmon, B. A., Lamb, R. C., \& Prince, T. A 1999, ApJ, 518, L99

Macomb, D. J., Fox, D. W., Lamb, R. C., \& Prince, T. A. 2003, ApJ, 584, L79

Maggi, P., Sturm, R., Haberl, F., \& Vasilopoulos, G. 2013, ATel, 5674, 1

Maggi, P., Sturm, R., Haberl, F., Vasilopoulos, G., \& Udalski, A. 2014, ATel, 5778, 1

Majid, W. A., Lamb, R. C., \& Macomb, D. J. 2004, ApJ, 609, 133

Maravelias, G., Zezas, A., Antoniou, V., \& Hatzidimitriou, D. 2014, MNRAS, 438, 2005

Marshall, F. E., Lochner, J. C., Santangelo, A., et al. 1998, IAU Circ., 6818, 1

Masetti, N., Parisi, P., Palazzi, E., et al. 2010, A\&A, 519, A96

Massey, P. 2002, ApJS, 141, 81

McBride, V. A., Coe, M. J., Bird, A. J., et al. 2007, MNRAS, 382, 743

McBride, V. A., Coe, M. J., Negueruela, I., Schurch, M. P. E., \& McGowan, K. E. 2008, MNRAS, 388, 1198

McBride, V. A., Bird, A. J., Coe, M. J., et al. 2010, MNRAS, 403, 709

McGowan, K. E., Coe, M. J., Schurch, M., et al. 2007, MNRAS, 376, 759

McGowan, K. E., Coe, M. J., Schurch, M. P. E., et al. 2008a, MNRAS, 384, 821

McGowan, K. E., Coe, M. J., Schurch, M. P. E., et al. 2008b, MNRAS, 383, 330

Meixner, M., Gordon, K. D., Indebetouw, R., et al. 2006, AJ, 132, 2268

Meyssonnier, N., \& Azzopardi, M. 1993, A\&AS, 102, 45

Murphy, M. T., \& Bessell, M. S. 2000, MNRAS, 311, 741

Nazé, Y., Hartwell, J. M., Stevens, I. R., et al. 2003a, ApJ, 586, 983

Nazé, Y., Rauw, G., Manfroid, J., Chu, Y.-H., \& Vreux, J.-M. 2003b, A\&A, 401, L13

Novara, G., La Palombara, N., Mereghetti, S., et al. 2011, A\&A, 532, A153
Raguzova, N. V., \& Popov, S. B. 2005, Astron. Astrophys. Trans., 24, 151 Rajoelimanana, A. F., Charles, P. A., \& Udalski, A. 2011a, ATel, 3154, 1 Rajoelimanana, A. F., Charles, P. A., \& Udalski, A. 2011b, MNRAS, 413, 1600 Reig, P. 2011, Ap\&SS, 332, 1

Reig, P., Fabregat, J., \& Coe, M. J. 1997, A\&A, 322, 193

Reig, P., Larionov, V., Negueruela, I., Arkharov, A. A., \& Kudryavtseva, N. A. 2007, A\&A, 462, 1081

Rivinius, T., Carciofi, A. C., \& Martayan, C. 2013, A\&ARv, 21, 69

Santangelo, A., Cusumano, G., Israel, G. L., et al. 1998, IAU Circ., 6818, 1

Sasaki, M., Haberl, F., \& Pietsch, W. 2000, A\&AS, 147, 75

Sasaki, M., Haberl, F., Keller, S., \& Pietsch, W. 2001, A\&A, 369, L29

Sasaki, M., Pietsch, W., \& Haberl, F. 2003, A\&A, 403, 901

Schmidtke, P. C., \& Cowley, A. P. 2005, AJ, 130, 2220

Schmidtke, P. C., \& Cowley, A. P. 2006, ATel, 716, 1

Schmidtke, P. C., \& Cowley, A. P. 2013, ATel, 5556, 1

Schmidtke, P. C., Cowley, A. P., Crane, J. D., et al. 1999, AJ, 117, 927

Schmidtke, P. C., Cowley, A. P., Levenson, L., \& Sweet, K. 2004, AJ, 127, 3388

Schmidtke, P. C., Cowley, A. P., \& Udalski, A. 2009, ATel, 1953, 1

Schmidtke, P. C., Cowley, A. P., \& Udalski, A. 2013a, MNRAS, 431, 252

Schmidtke, P. C., Cowley, A. P., \& Udalski, A. 2013b, ATel, 4936, 1

Schmidtke, P. C., Cowley, A. P., \& Udalski, A. 2014, ATel, 5776, 1

Schurch, M. P. E., Coe, M. J., McGowan, K. E., et al. 2007, MNRAS, 381, 1561

Schurch, M. P. E., Coe, M. J., McBride, V. A., et al. 2011, MNRAS, 412, 391

Shtykovskiy, P., \& Gilfanov, M. 2005, MNRAS, 362, 879

Skrutskie, M. F., Cutri, R. M., Stiening, R., et al. 2006, AJ, 131, 1163

Smith Neubig, M. M., \& Bruhweiler, F. C. 1997, AJ, 114, 1951

Stella, L., White, N. E., \& Rosner, R. 1986, ApJ, 308, 669

Stevens, J. B., Coe, M. J., \& Buckley, D. A. H. 1999, MNRAS, 309, 421

Sturm, R., Pietsch, W., Haberl, F., \& Immler, S. 2010, ATel, 2876, 1

Sturm, R., Haberl, F., Coe, M. J., et al. 2011a, A\&A, 527, A131

Sturm, R., Haberl, F., Pietsch, W., Immler, S., \& Udalski, A. 2011b, ATel, 3575, 1

Sturm, R., Haberl, F., Pietsch, W., \& Udalski, A. 2011c, ATel, 3761, 1

Sturm, R., Haberl, F., Pietsch, W., et al. 2012, A\&A, 537, A76

Sturm, R., Drašković, D., Filipović, M. D., et al. 2013a, A\&A, 558, A101

Sturm, R., Haberl, F., Oskinova, L. M., et al. 2013b, A\&A, 556, A139

Sturm, R., Haberl, F., Pietsch, W., et al. 2013c, A\&A, 558, A3

Sturm, R., Haberl, F., Pietsch, W., \& Udalski, A. 2013d, A\&A, 551, A96

Sturm, R., Haberl, F., Vasilopoulos, G., et al. 2014, MNRAS, 444, 3571

Townsend, L. J., Coe, M. J., Corbet, R. H. D., \& Hill, A. B. 2011a, MNRAS, 416, 1556

Townsend, L. J., Drave, S. P., Corbet, R. H. D., Coe, M. J., \& Bird, A. J. 2011b, ATel, 3311, 1

Tsujimoto, M., Imanishi, K., Yokogawa, J., \& Koyama, K. 1999, PASJ, 51, L21

Udalski, A., Szymanski, M., Kubiak, M., et al. 1998, Acta Astron., 48, 147

Wada, Q., Tsujimoto, M., \& Ebisawa, K. 2012, ATel, 4628, 1

Wada, Q., Tsujimoto, M., Ebisawa, K., \& Miller, E. D. 2013, PASJ, 65, L2

Wyrzykowski, L., Udalski, A., Kubiak, M., et al. 2004, Acta Astron., 54, 1

Yokogawa, J., \& Koyama, K. 1998a, IAU Circ., 7009, 3

Yokogawa, J., \& Koyama, K. 1998b, IAU Circ., 7028, 1

Yokogawa, J., Imanishi, K., Ueno, M., \& Koyama, K. 2000a, PASJ, 52, L73

Yokogawa, J., Torii, K., Imanishi, K., \& Koyama, K. 2000b, PASJ, 52, L37

Yokogawa, J., Torii, K., Kohmura, T., Imanishi, K., \& Koyama, K. 2000c, PASJ, $52, \mathrm{~L} 53$

Yokogawa, J., Imanishi, K., Tsujimoto, M., Koyama, K., \& Nishiuchi, M. 2003, PASJ, 55, 161

Zaritsky, D., Harris, J., Thompson, I. B., Grebel, E. K., \& Massey, P. 2002, AJ, 123,855 


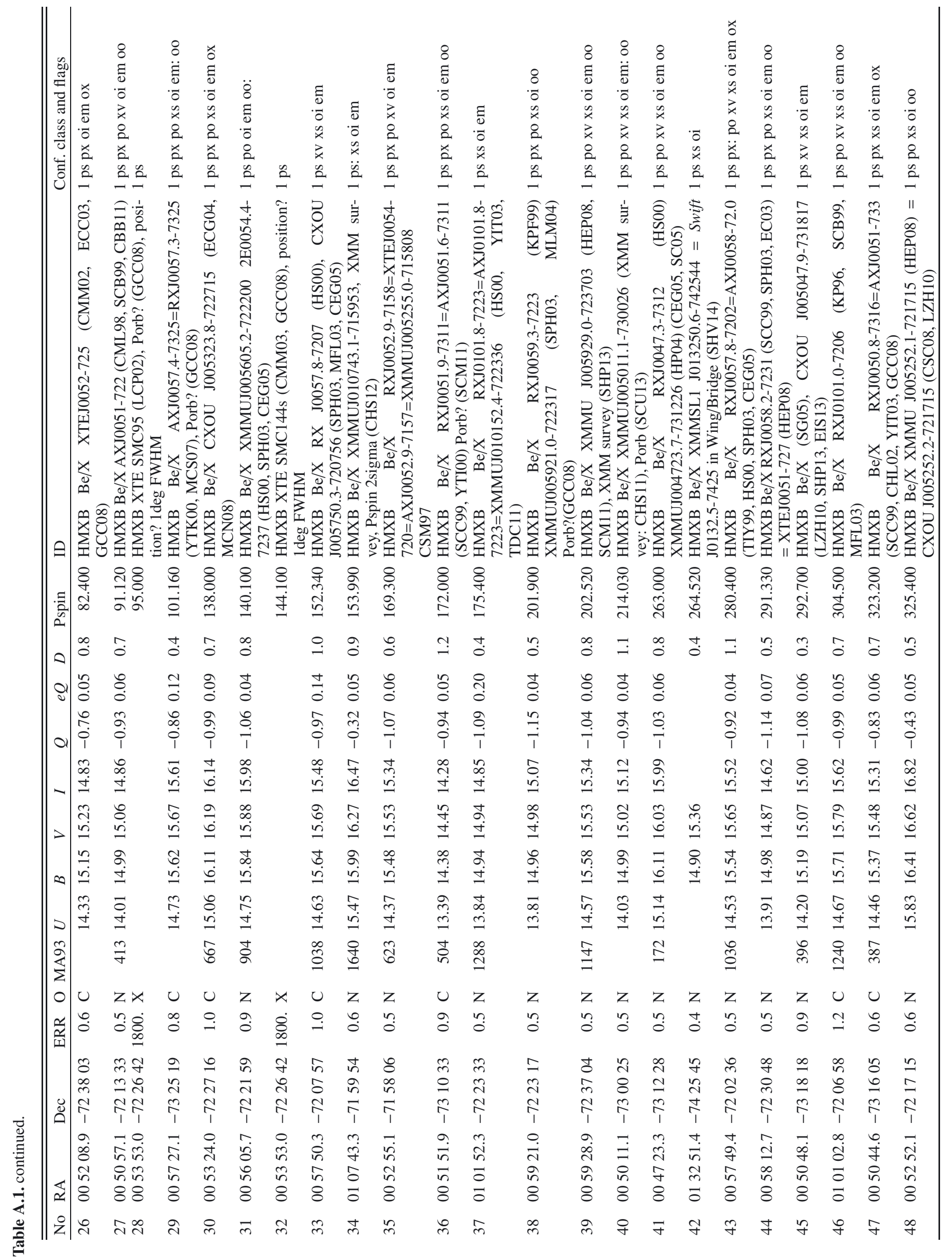




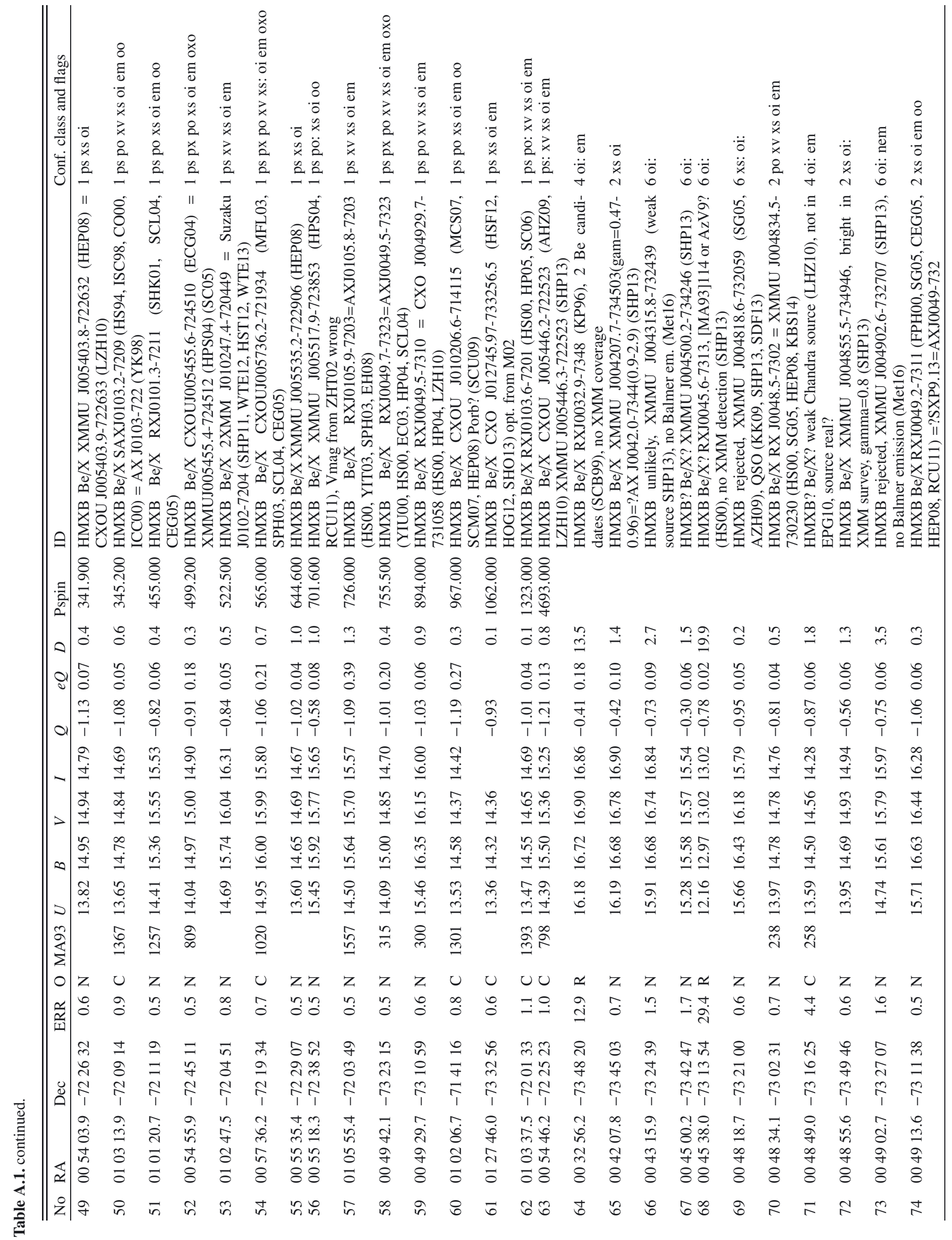




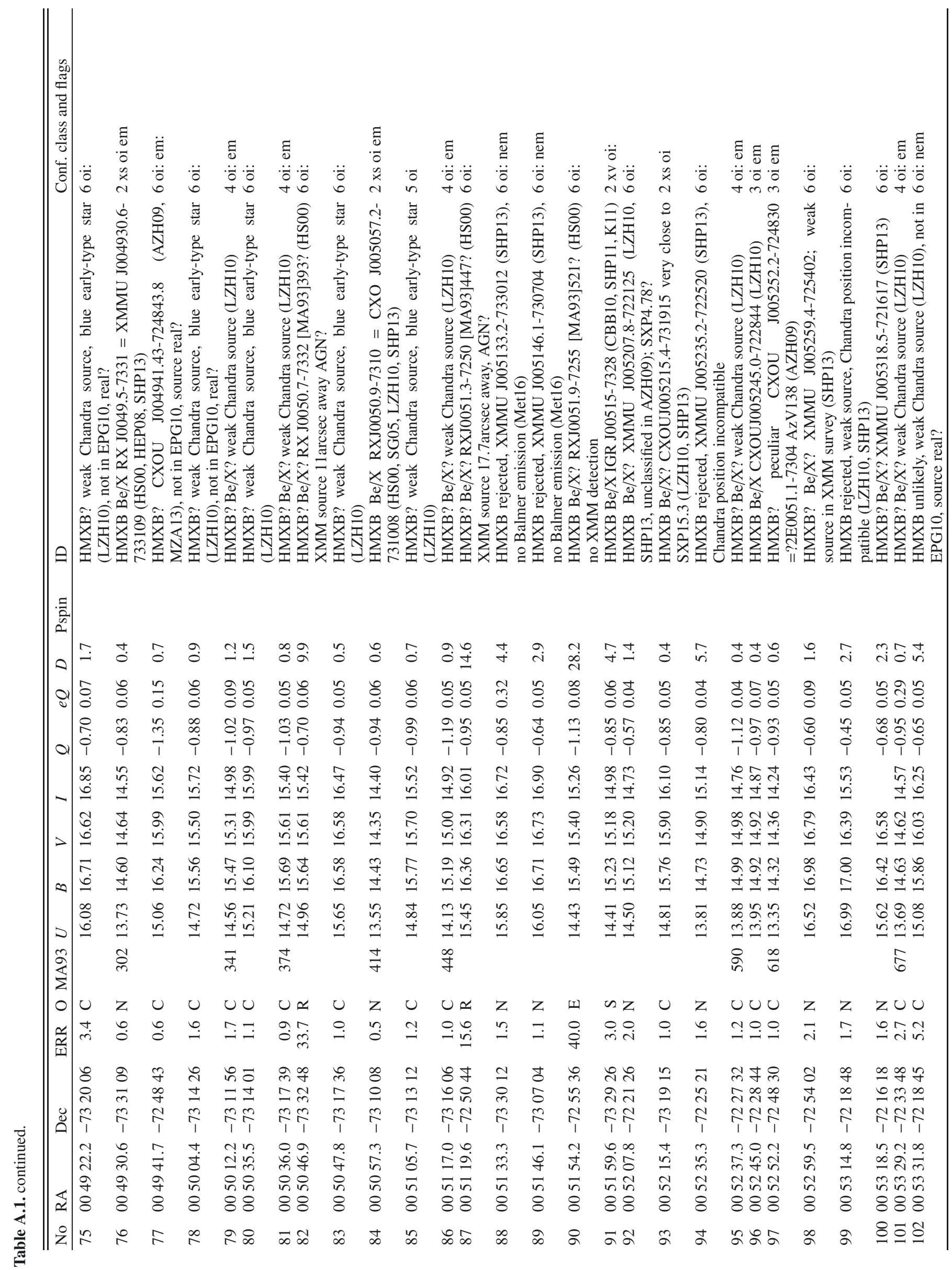


F. Haberl and R. Sturm: High-mass X-ray binaries in the SMC

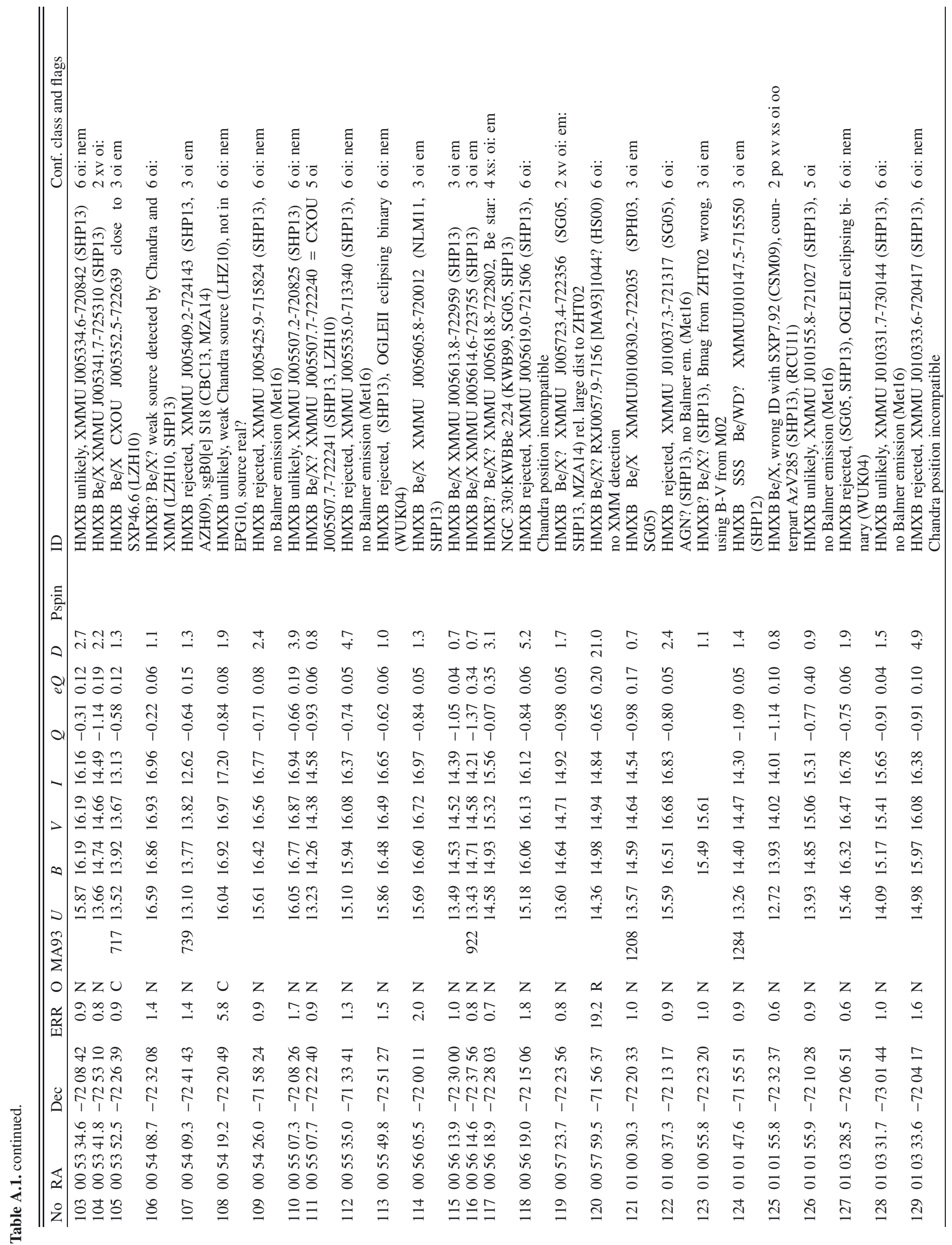




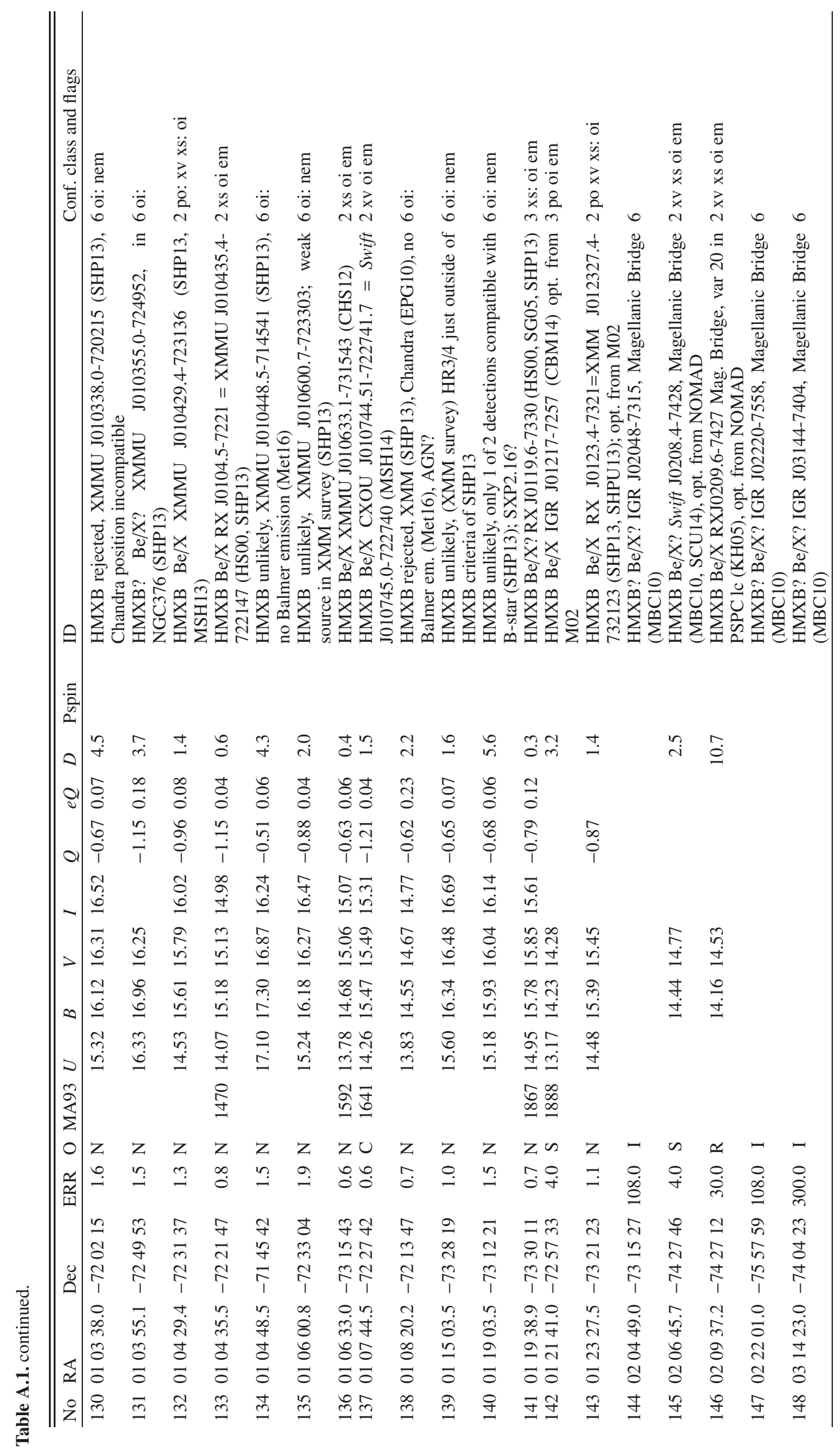


Table A.2. Key references.

\begin{tabular}{|c|c|c|c|}
\hline $\operatorname{Code}^{a}$ & Reference & Code $^{a}$ & Reference \\
\hline AHZ09 & Antoniou et al. (2009a) & AZH09 & Antoniou et al. (2009b) \\
\hline BCS01 & Buckley et al. (2001) & BLK10 & Bonanos et al. (2010) \\
\hline CBB10 & Coe et al. (2010) & CBB11 & Coe et al. (2011a) \\
\hline CBB15 & Coe et al. (2015) & $\mathrm{CBC} 13$ & Clark et al. (2013) \\
\hline CBK13 & Coe et al. (2013a) & CBM13 & Coe et al. (2013b) \\
\hline CBM14 & Coe et al. (2014) & CCM08 & Corbet et al. (2008) \\
\hline CEG05 & Coe et al. (2005) & CHL02 & Coe et al. (2002) \\
\hline CHS11 & Coe et al. (2011b) & CHS12 & Coe et al. (2012) \\
\hline CLC98 & Chakrabarty et al. (1998a) & CML98 & Corbet et al. (1998) \\
\hline CMM02 & Corbet et al. (2002) & CMM03 & Corbet et al. (2003) \\
\hline CNC01 & Covino et al. (2001) & $\mathrm{COOO}$ & Coe \& Orosz (2000) \\
\hline CRW97 & Clark et al. (1997) & CSC08 & Schmidtke et al. (1999) \\
\hline CSM97 & Cowley et al. (1997) & CSM09 & Coe et al. (2009) \\
\hline СТО98 & Chakrabarty et al. (1998b) & EC03 & Edge \& Coe (2003) \\
\hline ECC03 & Edge et al. (2003) & ECG04 & Edge et al. (2004a) \\
\hline EH08 & Eger \& Haberl (2008b) & EHI04 & Evans et al. (2004) \\
\hline EIS13 & Esposito et al. (2013) & ELS06 & Evans et al. (2006) \\
\hline EPG10 & Evans et al. (2010) & FPHOO & Filipović et al. (2000b) \\
\hline GCC08 & Galache et al. (2008) & HEP08 & Haberl et al. (2008) \\
\hline HPK07 & Haberl et al. (2007) & HP05 & Haberl \& Pietsch (2005) \\
\hline HPS04 & Haberl et al. (2004) & HOG12 & Hénault-Brunet et al. (2012) \\
\hline HP04 & Haberl \& Pietsch (2004) & HS94 & Hughes \& Smith (1994) \\
\hline HSOO & Haberl \& Sasaki (2000) & HSF12 & Haberl et al. (2012a) \\
\hline HST12 & Haberl et al. (2012b) & ICC00 & Israel et al. (2000) \\
\hline IED13 & Israel et al. (2013) & ISA97 & Israel et al. (1997) \\
\hline ISC98 & Israel et al. (1998) & ISC99 & Israel et al. (1999) \\
\hline K11 & Kennea (2011) & KBS14 & Kourniotis et al. (2014) \\
\hline KH05 & Kahabka \& Hilker (2005) & KPF99 & Kahabka et al. (1999) \\
\hline KWB99 & Keller et al. (1999) & $\mathrm{LCP} 02$ & Laycock et al. (2002) \\
\hline LMP02 & Lamb et al. (2002) & LPM99 & Lamb et al. (1999) \\
\hline LZH10 & Laycock et al. (2010) & MA93 & Meyssonnier \& Azzopardi (1993) \\
\hline M02 & Massey (2002) & MBC10 & McBride et al. (2010) \\
\hline MCB07 & McBride et al. (2007) & MCN08 & McBride et al. (2008) \\
\hline MCS07 & McGowan et al. (2007) & MCS08 & McGowan et al. (2008a) \\
\hline Met16 & McBride et al. (in prep.) & MFH99 & Macomb et al. (1999) \\
\hline MFL03 & Macomb et al. (2003) & MLM04 & Majid et al. (2004) \\
\hline MLS98 & Marshall et al. (1998) & MPP10 & Masetti et al. (2010) \\
\hline MSH13 & Maggi et al. (2013) & MSH14 & Maggi et al. (2014) \\
\hline MZA14 & Maravelias et al. (2014) & NLM11 & Novara et al. (2011) \\
\hline NHS03 & Nazé et al. (2003a) & RCU11 & Rajoelimanana et al. (2011b) \\
\hline $\mathrm{SC} 05$ & Schmidtke \& Cowley (2005) & SC06 & Schmidtke \& Cowley (2006) \\
\hline SC13 & Schmidtke \& Cowley (2013) & SCB99 & Stevens et al. (1999) \\
\hline SCC99 & Schmidtke et al. (1999) & SCI98 & Santangelo et al. (1998) \\
\hline SCL04 & Schmidtke et al. (2004) & SCM07 & Schurch et al. (2007) \\
\hline SCM11 & Schurch et al. (2011) & SCU09 & Schmidtke et al. (2009) \\
\hline SCU13 & Schmidtke et al. (2013b) & SCU14 & Schmidtke et al. (2014) \\
\hline SDF13 & Sturm et al. (2013a) & SG05 & Shtykovskiy \& Gilfanov (2005) \\
\hline SHC11 & Sturm et al. (2011a) & SHK01 & Sasaki et al. (2001) \\
\hline SHO13 & Sturm et al. (2013b) & SHP11 & Sturm et al. (2011c) \\
\hline SHP12 & Sturm et al. (2012) & SHP13 & Sturm et al. (2013c) \\
\hline SHPU13 & Sturm et al. (2013d) & SHV14 & Sturm et al. (2014) \\
\hline SPH03 & Sasaki et al. (2003) & SPH10 & Sturm et al. (2010) \\
\hline SHP11 & Sturm et al. (2011b) & TDC11 & Townsend et al. (2011b) \\
\hline TIY99 & Tsujimoto et al. (1999) & WTE12 & Wada et al. (2012) \\
\hline WTE13 & Wada et al. (2013) & WUK04 & Wyrzykowski et al. (2004) \\
\hline YIT03 & Yokogawa et al. (2003) & YIU00 & Yokogawa et al. (2000a) \\
\hline YK98 & Yokogawa \& Koyama (1998a) & YTI00 & Yokogawa et al. (2000b) \\
\hline YTK00 & Yokogawa et al. (2000c) & ZHT02 & Zaritsky et al. (2002) \\
\hline
\end{tabular}

Notes. ${ }^{(a)}$ As used in the comment column of Table A.1. 